\title{
Global burden of HIV/AIDS in 2004 resulting from non-adherence to medication regimes and alcohol-attributable non-adherence to medication regimes
}

\author{
Kevin D. Shield ${ }^{1,2}$, Paul A. Shuper ${ }^{1,3}$, Gerrit Gmel ${ }^{1}$, and Jürgen Rehm ${ }^{1,2,3,4,5}$ \\ ${ }^{1}$ Centre for Addiction and Mental Health, Toronto, Canada \\ ${ }^{2}$ Institute of Medical Science, University of Toronto, Toronto, Canada \\ ${ }^{3}$ Department of Psychology, University of Toronto, Toronto, Canada \\ ${ }^{4}$ Dalla Lana School of Public Health, University of Toronto, Toronto, Canada \\ ${ }^{5}$ Institute for Clinical Psychology and Psychotherapy, Technische Universität Dresden, Dresden, Germany
}

\section{Abstract}

Aims: Using novel methodology, this article aims to quantify the number of HIV/AIDS-related deaths and the potential years of life lost (PYLL) in 2004 resulting from non-adherence to antiretroviral treatment (ART) and non-adherence to ART due to alcohol consumption.

Design: HIV/AIDS-related deaths and PYLL attributable to non-adherence to ART and attributable specifically to non-adherence due to alcohol consumption were calculated using attributable fractions. These fractions were based on new risk modeling methodology, which combines estimates of the mortality of those people currently adhering to ART, the mortality attributable to non-adherence to ART, and the proportion of those people not adhering to ART because of alcohol consumption.

Measurements: Data on alcohol indicators were obtained from the Comparative Risk Assessment study, data on deaths and PYLL were obtained from the World Health Organization, and ART indicators were obtained from UNAIDS.

Findings: In 2004, for people 15 years of age and older, 67,000 (95\% CI: 62,000-72,000) deaths and 1,608,000 (95\% CI: 1,491,000-1,725,000) PYLL were caused by non-adherence to ART, of which 8,000 (95\% CI: 3,000-13,000) HIV/AIDS-related deaths and 187,000 (95\% CI: 70,000-304,000) HIV/AIDS-related PYLL were attributable to alcohol consumption.

Conclusions: As the burden of disease for HIV/AIDS-related deaths attributable to non-adherence to ART and attributable to alcohol consumption is non-trivial, additional research is required to examine the effectiveness of different interventions aimed at reducing alcohol consumption among people with HIV/AIDS and at increasing adherence to ART among both drinkers and nondrinkers.

The global burden of HIV/AIDS is substantial, with an estimated 2.8 million new HIV infections, 2.0 million HIVattributable deaths, and 58.5 million disability-adjusted years of life lost (a combination of years of life lost due to premature mortality and years lived with disability) due to HIV/AIDS in 2004 (World Health Organization [WHO], 2008). During the 2004 Global Burden of Disease (GBD) study, evidence was not available for a causal relationship between alcohol consumption and the probability of becoming infected by HIV and worsening of disease course for people with HIV/AIDS, so previous iterations of the Comparative Risk Assessment (CRA) study (a study that compares the impact of various modifiable risk factors on death and disability) did not include HIV/AIDS in the estimates of the alcohol-attributable burden of disease (Rehm et al., 2010; WHO, 2009).
This lack of causal evidence was in part attributable to the lack of research on how personality factors confound and/or mediate the relationship between alcohol consumption and the probability of HIV/AIDS (Dingle \& Oei, 1997; Shuper et al., 2010; Stall \& Leigh, 1994). To control for these personality factors Rehm, Shield, Joharchi, and Shuper (2012) performed a meta-analysis, combining results from different randomized controlled studies. This study found a causal relationship between alcohol consumption and unsafe sex, which leads to an increased probability of acquiring HIV through sexual transmission (see Rehm et al., 2012, for the causal relationship between alcohol consumption and unsafe sex, and Baliunas, Rehm, Irving, \& Shuper, 2010, and Fisher, Bang, \& Kapiga, 2007, for the magnitude of the association). In addition to this causal association, alcohol consumption has

Correspondence: Kevin D. Shield, Centre for Addiction and Mental Health, 33 Russell Street, Toronto, ON, M5S 2S1, Canada

Phone: +1 647-971 6175; Fax: +1 416-260-4146. E-mail: kevin.shield@utoronto.ca

Competing interests: The authors have declared that no competing interests exist.

Financial support: Support to CAMH for salary of scientist and infrastructure is provided by the Ontario Ministry of Health and Long-Term Care.

Keywords: alcohol consumption, HIV, antiretroviral therapy, adherence, mortality, potential years of life lost 
been shown to affect susceptibility to HIV infection by weakening the immune system (Baliunas et al., 2010; Braithwaite et al., 2005; Hahn \& Samet, 2010; Neuman, Monteiro, \& Rehm, 2006; Shuper, Joharchi, Irving, \& Rehm, 2009; Shuper et al., 2010). Although a causal association exists between alcohol consumption and acquiring HIV/AIDs through sexual transmission, estimation of the burden of HIV/AIDS due to alcoholattributable acquired HIV infections is currently not possible as the methodology to calculate this burden does not exist.

In addition to a causal relationship between alcohol consumption and acquiring HIV though sexual transmission, there is sufficient causal evidence that alcohol worsens the disease course of HIV/AIDS and can subsequently increase the likelihood of mortality, especially by impacting adherence to antiretroviral treatment (ART) (Azar, Springer, Meyer, \& Altice, 2010; Braithwaite et al., 2007; Gmel, Shield, \& Rehm, 2010; Hendershot, Stoner, Pantalone, \& Simoni, 2009). Specifically in a meta-analysis of alcohol and ART adherence, individuals who indicated that they had consumed alcohol within the past year were approximately $60 \%(O R=0.60$, CI: $0.53-0.69)$ as likely to be adherent to ART as were non-drinkers. Drinkers whose alcohol consumption patterns were problematic were approximately $47 \%(O R=0.47$, CI: $0.41-0.55)$ as likely to be adherent to ART as were non-problematic drinkers or abstainers (Hendershot et al., 2009). This association is also supported by previous research, which has found that ART doses were missed most frequently among binge drinkers and that the relative frequency of these missed doses occurred on binge drinking days (Braithwaite et al., 2005).

Given the association between alcohol consumption and non-adherence to ART, and given the significant impact of ART non-adherence on mortality (e.g., Garcia de Olalla et al., 2002; Lima et al., 2008; Lima et al., 2009; Nachega et al., 2006) it is the objective of this paper to estimate the deaths and potential years of life lost (PYLL) caused by HIV/AIDS due to non-adherence to ART and nonadherence to ART attributable to alcohol consumption.

\section{Method}

The methodology employed in this investigation consisted of the following two main steps: (1) calculation of nonadherence attributable fractions (NAdAFs) and alcoholattributable fractions (AAFs) by age, sex, and region; and (2) application of the NAdAFs and AAFs to region-, age-, and sex-specific mortality, and to region-, age-, and sexspecific PYLL.

\section{Definition of regions and population data}

The GBD regions (2005) are based on geography, child and adult mortality, and major causes of death (Institute for Health Metrics and Evaluation [IHME], 2010; see http:// www.globalburden.org/gbdops.html for the countries included in each region). Population estimates by country for 2004 were based on data obtained from the 2008 revisions of the United Nations Population Division (2010).

\section{Definition of age categories}

Three age categories were used in the CRA study: 15-34, $35-64$, and 65 years of age or older. Ages were clustered so as to be comparable to the 2005 GBD study (IHME, 2010).

\section{Measures of alcohol consumption}

Data on alcohol use for 2005 were compiled for the GBD regions based on drinking status. Current drinkers were defined as people who had consumed at least one drink in the past year, and current abstainers were defined as people who had not consumed alcohol within the past year. Data on drinking status were obtained from large representative population surveys taken in the 2000s (WHO, 2011). The prevalence of heavy drinkers, defined as those consuming on average 40 or more grams of alcohol per day for women (3.33 standard drinks or more per day) and 60 or more grams of alcohol per day for men (5 standard drinks or more) was calculated based on methods developed by Kehoe and colleagues, whereby the distribution of average daily consumption of alcohol is estimated based on $80 \%$ of the mean alcohol consumption for men and women (allowing 20\% for alcohol not consumed due to spillage, breakage, and waste, and to account for the underreporting of alcohol consumption commonly seen in surveys (Kehoe et al., 2012; Shield \& Rehm, 2012).

\section{Measures of the prevalence of antiretroviral therapy}

Country data on the proportion of people who were in need of ART and who were also receiving ART were obtained for 2005 from the 2006 Report on the Global AIDS Epidemic (UNAIDS, 2006). Regional data were then estimated based on a population-weighted average. The prevalence of those in need of antiretroviral treatment who were also receiving treatment, by region, is outlined in Appendix Table 1.

The prevalence of people adhering to antiretroviral therapy was estimated to be $40.1 \%$ (95\% CI: 36.9\%-43.3\%), provided by Lima and colleagues (2008). This estimate was similar to two other estimates of $47.6 \%$ and $31.8 \%$ for adherence rates of less than $80 \%$ and less than $90 \%$ respectively (Garcia de Olalla et al., 2002; Nachega et al., 2006).

\section{Measures of risk}

Multiple risk measures were used in calculating the association between alcohol consumption and HIV/AIDSrelated mortality from non-adherence to ART. To characterize the association between alcohol consumption and ART adherence, the odds ratio (OR) of 0.60 (95\% CI: 0.53-0.69), comparing the odds of adherence by drinkers versus non-drinkers, was based on results from a metaanalysis performed by Hendershot and colleagues (2009). To calculate the AAF of HIV/AIDS-related mortality due 
to heavy consumption of alcohol, we performed a sensitivity analysis where people who were considered heavy consumers of alcohol had an OR of 0.47 (95\% CI: 0.41-0.55) (in this sensitivity analysis, as no alternative existed, the above-noted $O R$ of 0.60 was used for nonheavy drinkers (Hendershot et al., 2009)). Since no metaanalyses exist of the association between non-adherence and mortality, we used a hazards ratio (HR) for mortality of 3.13 (95\% CI: 1.95-5.05) for people not adhering to ART compared to people who adhere to their ART regime (where non-adherence is defined as adhering to ART less than $95 \%$ of the time (Lima et al., 2009)). This HR was similar to findings from two other studies, which observed HRs of 3.2 and 3.9 for adherence rates of less than $80 \%$ and less than $90 \%$ respectively (Garcia de Olalla et al., 2002; Nachega et al., 2006) for the mortality of people on ART compared to those who were not on ART.

Step 1: Calculation of the NAdAFs and AAFs by sex and by alcohol consumption

The AAF for mortality due to non-adherence to ART was calculated using three composite measures: (1) the adherence attributable fraction (AdAF), (2) the nonadherence due to alcohol attributable fraction (NAAAF), and (3) the proportion of deaths of people undergoing treatment (PDT).

AAFs were calculated as follows:

\section{$A A F=A d A F^{*} N A A A F^{*} P D T$}

NAdAFs were calculated using two of the above-noted composite measures as follows:

NAdAFs $=A d A F * P D T$

The AdAF, defined as the fraction of HIV/AIDS-related deaths attributable to non-adherence to antiretroviral therapy, using a definition of non-adherence as adherence to ART less than $95 \%$ of the time, was calculated as follows:

$$
A d A F=\frac{P_{a d h e r}+P_{n a} R R_{n a}-1}{P_{a d h e r}+P_{n a} R R_{n a}}
$$

where $P_{a d h e r}$ is the proportion of people adhering to ART more than $95 \%$ of the time, $P_{n a}$ is the proportion of people adhering to treatment less than $95 \%$ of the time, and $\mathrm{RR}_{\mathrm{na}}$ is the relative risk of mortality for those people not adhering to medication compared to those people who follow a treatment regime.

The NAAAF, defined as the proportion of non-adherence due to alcohol consumption, was calculated as follows for all HIV/AIDS-related deaths due to alcohol consumption:

$$
N A A A F=\frac{P_{a b s}+P_{d r i n k} R R_{d r i n k}-1}{P_{a b s}+P_{d r i n k} R R_{d r i n k}}
$$

and as follows for all HIV/AIDS-related deaths due to heavy alcohol consumption:

$$
N A A A F=\frac{P_{a b s}+P_{H_{\_} d r i n k e r} R R_{H_{\_} d r i n k e r}+P_{N H_{\_} d r i n k e r}-1}{P_{a b s}+P_{H_{-} d r i n k e r} R R_{H_{-} d r i n k e r}+P_{N H_{-} d r i n k e r} R R_{\text {drinker }}}
$$

where $P_{\text {drink }}$ is the proportion of current drinkers, $P_{\text {abs }}$ represents the current abstainers among the antiretroviraltreated HIV population, $P_{H_{-} \text {drinker }}$ represents the prevalence of heavy drinkers in the population, $P_{N H \_d r i n k e r}$ represents the prevalence of heavy drinkers, and $R R_{\text {drink }}$ represents the relative risk of not adhering to ART for a current drinker compared to a current abstainer.

Defined as PDT, the proportion of HIV/AIDS-related deaths of those people currently under treatment compared to those who are under treatment was calculated as follows:

$$
P D T=\frac{P_{\text {treat }}}{P_{\text {treat }}+H R_{\text {nontreat }} P_{\text {nontreat }}}
$$

where $P_{\text {treat }}$ is the proportion of HIV-infected people who are in need of ART and who are receiving it, $P_{\text {nontreat }}$ is the proportion of HIV-infected people who are in need of ART and who are not receiving it, and $H R_{\text {nontreat }}$ is the hazards ratio for mortality of those people not adhering to ART compared to those who are adhering to ART.

\section{Estimation of the variances of the NAdAFs and AAFs}

The variances of the NAdAFs and AAFs were estimated by using the variance of each parameter making up the final expression. Specifically, the variances of AdAF, NAAAF, and PDT were first estimated using the variances of the parameters used in calculating each of these measures. Next, the variance estimates of AdAF and PDT were used to estimate the variances of NAdAFs, and the variance estimates AdAF, NAAAF, and PDT were used to estimate the variances of the AAFs for each region by age and by sex. For the formulas used to calculate the variance of the AAFs, see Gmel et al., 2010.

Step 2: Application of the NAdAFs and AAFs to region-, age-, and sex-specific mortality data, and to region-, age-, and sex-specific PYLL data

This step required multiplying the sex-, age-, and regionspecific NAdAFs and AAFs by mortality data and by PYLL data, respectively.

\section{Estimates of mortality and morbidity}

To quantify the burden of disease attributable to alcohol consumption we used an event-based measure (mortality) and time-based measures (PYLL). Comprehensive revision estimates of mortality and PYLL for 2004 were provided by WHO (2008) for the 160 GBD disease and injury 
Table 1a

Deaths attributable to HIV/AIDS from not adhering to antiretroviral therapy by Global Burden of Disease region for 2004 (Men)

\begin{tabular}{|c|c|c|c|c|c|c|c|c|c|}
\hline \multirow[b]{2}{*}{$\begin{array}{l}\text { Global Burden of } \\
\text { Disease region }\end{array}$} & \multicolumn{3}{|c|}{$15-34$ years } & \multicolumn{3}{|c|}{ 35-64 years } & \multicolumn{3}{|c|}{$65+$ years } \\
\hline & $\begin{array}{c}\text { Point } \\
\text { estimate }\end{array}$ & $\begin{array}{c}\text { Lower } \\
\text { estimate }\end{array}$ & $\begin{array}{c}\text { Upper } \\
\text { estimate }\end{array}$ & $\begin{array}{c}\text { Point } \\
\text { estimate }\end{array}$ & $\begin{array}{c}\text { Lower } \\
\text { estimate }\end{array}$ & $\begin{array}{c}\text { Upper } \\
\text { estimate }\end{array}$ & $\begin{array}{c}\text { Point } \\
\text { estimate }\end{array}$ & $\begin{array}{c}\text { Lower } \\
\text { estimate }\end{array}$ & $\begin{array}{c}\begin{array}{c}\text { Upper } \\
\text { estimate }\end{array} \\
\end{array}$ \\
\hline $\begin{array}{l}\text { Asia, Pacific } \\
\text { [High Income] }\end{array}$ & 12 & 11 & 14 & 32 & 28 & 36 & 3 & 3 & 3 \\
\hline Asia, Central & 6 & 5 & 7 & 6 & 5 & 7 & 0 & 0 & 0 \\
\hline Asia, East & 304 & 266 & 342 & 488 & 425 & 551 & 6 & 6 & 7 \\
\hline Asia, South & 485 & 464 & 505 & 419 & 394 & 444 & 4 & 4 & 5 \\
\hline Asia, Southeast & 1,686 & 1,567 & 1,805 & 2,318 & 2,179 & 2,457 & 31 & 29 & 32 \\
\hline Australasia & 4 & 3 & 4 & 13 & 11 & 14 & 2 & 2 & 2 \\
\hline Caribbean & 417 & 368 & 466 & 801 & 709 & 892 & 28 & 25 & 30 \\
\hline Europe, Central & 31 & 28 & 35 & 37 & 32 & 41 & 2 & 2 & 3 \\
\hline Europe, Eastern & 103 & 89 & 117 & 138 & 119 & 157 & 2 & 2 & 2 \\
\hline Europe, Western & 247 & 216 & 278 & 702 & 610 & 794 & 65 & 58 & 73 \\
\hline $\begin{array}{l}\text { Latin America, } \\
\text { Andean }\end{array}$ & 207 & 183 & 231 & 265 & 232 & 299 & 4 & 4 & 4 \\
\hline $\begin{array}{l}\text { Latin America, } \\
\text { Central }\end{array}$ & 1,042 & 943 & 1,141 & 1,412 & 1,258 & 1,566 & 45 & 41 & 49 \\
\hline $\begin{array}{l}\text { Latin America, } \\
\text { Southern }\end{array}$ & 155 & 135 & 175 & 298 & 261 & 335 & 18 & 16 & 20 \\
\hline $\begin{array}{l}\text { Latin America, } \\
\text { Tropical }\end{array}$ & 1,127 & 1,007 & 1,248 & 2,012 & 1,831 & 2,194 & 84 & 79 & 89 \\
\hline $\begin{array}{l}\text { Northern Africa / } \\
\text { Middle East }\end{array}$ & 51 & 49 & 52 & 57 & 55 & 60 & 1 & 1 & 1 \\
\hline $\begin{array}{l}\text { North America } \\
\text { [High Income] }\end{array}$ & 411 & 362 & 460 & 1,656 & 1,465 & 1,847 & 117 & 106 & 128 \\
\hline Oceania & 7 & 6 & 8 & 13 & 12 & 15 & 0 & 0 & 0 \\
\hline $\begin{array}{l}\text { Sub-Saharan } \\
\text { Africa, Central }\end{array}$ & 84 & 75 & 94 & 165 & 146 & 183 & 4 & 4 & 4 \\
\hline $\begin{array}{l}\text { Sub-Saharan } \\
\text { Africa, East }\end{array}$ & 1,974 & 1,852 & 2,097 & 4,490 & 4,106 & 4,873 & 162 & 148 & 175 \\
\hline $\begin{array}{l}\text { Sub-Saharan } \\
\text { Africa, Southern }\end{array}$ & 2,912 & 2,672 & 3,152 & 6,556 & 6,015 & 7,096 & 163 & 152 & 174 \\
\hline $\begin{array}{l}\text { Sub-Saharan } \\
\text { Africa, Western }\end{array}$ & 902 & 829 & 976 & 2,019 & 1,810 & 2,227 & 68 & 62 & 74 \\
\hline World & 12,167 & 11,127 & 13,207 & 23,896 & 21,703 & 26,089 & 809 & 742 & 875 \\
\hline
\end{tabular}

categories. Methods to estimate the mortality and the PYLL in the GBD project are described elsewhere (Lopez, Mathers, Ezzati, Jamison, \& Murray, 2006a, 2006b). Estimates of mortality and of PYLL were available for each country by age and sex and were used to calculate region estimates. This meant that for each region, sex-, injury-, and consumption-specific AAFs were applied to sex- and injury-specific outcome data.

We used the 2004 estimate as it is the last estimate from the GBD study, and thus has been processed for coherence with other causes of death and consistency, and corrected for comparability between regions (WHO, 2008; see also Lopez et al., 2006b, for a general description of GBD methodology).
All statistics and analyses were performed using $\mathrm{R}$ version 2.11.1.

\section{Results}

The mortality and PYLL caused by non-adherence to ART are outlined in Tables $1 \mathrm{a}$ and $1 \mathrm{~b}$, and Tables 2a and $2 \mathrm{~b}$ respectively. In 2004 an estimated 67,386 (95\% CI: 62,391-72,380) deaths and 1,680,155 (95\% CI: 1,491,0001,725,000) PYLL were caused by HIV/AIDS-related mortality due to non-adherence to ART for people 15 years of age and older, representing 3.30\% (95\% CI: 3.06\%$3.55 \%$ ) of all deaths and 3.07\% (95\% CI: $2.84 \%-3.29 \%)$ of all PYLL caused by HIV/AIDS. Men had a slightly higher rate of HIV/AIDS-related deaths and PYLL due to nonadherence to ART, with the age groups of 15-34 and 35-64 
Table 1b

Deaths attributable to HIV/AIDS from not adhering to antiretroviral therapy by Global Burden of Disease region for 2004 (Women)

\begin{tabular}{|c|c|c|c|c|c|c|c|c|c|}
\hline \multirow[b]{2}{*}{$\begin{array}{l}\text { Global Burden of } \\
\text { Disease region }\end{array}$} & \multicolumn{3}{|c|}{ 15-34 years } & \multicolumn{3}{|c|}{ 35-64 years } & \multicolumn{3}{|c|}{$65+$ years } \\
\hline & $\begin{array}{c}\text { Point } \\
\text { estimate }\end{array}$ & $\begin{array}{c}\text { Lower } \\
\text { estimate }\end{array}$ & $\begin{array}{c}\text { Upper } \\
\text { estimate }\end{array}$ & $\begin{array}{c}\begin{array}{c}\text { Point } \\
\text { estimate }\end{array} \\
\end{array}$ & $\begin{array}{c}\text { Lower } \\
\text { estimate }\end{array}$ & $\begin{array}{c}\text { Upper } \\
\text { estimate }\end{array}$ & $\begin{array}{c}\text { Point } \\
\text { estimate }\end{array}$ & $\begin{array}{c}\text { Lower } \\
\text { estimate }\end{array}$ & $\begin{array}{c}\text { Upper } \\
\text { estimate }\end{array}$ \\
\hline $\begin{array}{l}\text { Asia, Pacific } \\
\text { [High Income] }\end{array}$ & 3 & 3 & 4 & 4 & 3 & 4 & 1 & 1 & 1 \\
\hline Asia, Central & 1 & 1 & 1 & 1 & 1 & 1 & 0 & 0 & 0 \\
\hline Asia, East & 87 & 80 & 94 & 107 & 99 & 116 & 3 & 2 & 3 \\
\hline Asia, South & 174 & 172 & 177 & 107 & 105 & 109 & 3 & 3 & 3 \\
\hline Asia, Southeast & 533 & 518 & 547 & 565 & 554 & 577 & 16 & 16 & 17 \\
\hline Australasia & 1 & 1 & 1 & 1 & 1 & 1 & 0 & 0 & 0 \\
\hline Caribbean & 278 & 253 & 304 & 357 & 334 & 379 & 12 & 12 & 13 \\
\hline Europe, Central & 19 & 17 & 21 & 11 & 10 & 12 & 0 & 0 & 0 \\
\hline Europe, Eastern & 29 & 26 & 32 & 32 & 28 & 35 & 1 & 1 & 1 \\
\hline Europe, Western & 80 & 70 & 90 & 171 & 149 & 192 & 14 & 13 & 15 \\
\hline $\begin{array}{l}\text { Latin America, } \\
\text { Andean }\end{array}$ & 63 & 57 & 68 & 62 & 56 & 68 & 2 & 2 & 2 \\
\hline $\begin{array}{l}\text { Latin America, } \\
\text { Central }\end{array}$ & 320 & 296 & 343 & 333 & 309 & 357 & 11 & 10 & 12 \\
\hline $\begin{array}{l}\text { Latin America, } \\
\text { Southern }\end{array}$ & 54 & 47 & 60 & 75 & 67 & 82 & 4 & 3 & 4 \\
\hline $\begin{array}{l}\text { Latin America, } \\
\text { Tropical }\end{array}$ & 625 & 570 & 680 & 997 & 925 & 1,068 & 47 & 45 & 49 \\
\hline $\begin{array}{l}\text { Northern Africa / } \\
\text { Middle East }\end{array}$ & 15 & 14 & 15 & 13 & 13 & 13 & 0 & 0 & 0 \\
\hline $\begin{array}{l}\text { North America } \\
\text { [High Income] }\end{array}$ & 194 & 172 & 216 & 578 & 517 & 639 & 37 & 35 & 40 \\
\hline Oceania & 4 & 3 & 4 & 5 & 4 & 5 & 0 & 0 & 0 \\
\hline $\begin{array}{l}\text { Sub-Saharan } \\
\text { Africa, Central }\end{array}$ & 154 & 141 & 166 & 206 & 191 & 221 & 5 & 5 & 6 \\
\hline $\begin{array}{l}\text { Sub-Saharan } \\
\text { Africa, East }\end{array}$ & 3,615 & 3,447 & 3,782 & 5,367 & 5,031 & 5,703 & 114 & 107 & 120 \\
\hline $\begin{array}{l}\text { Sub-Saharan } \\
\text { Africa, Southern }\end{array}$ & 4,478 & 4,303 & 4,653 & 6,599 & 6,302 & 6,897 & 157 & 152 & 163 \\
\hline $\begin{array}{l}\text { Sub-Saharan } \\
\text { Africa, Western }\end{array}$ & 1,524 & 1,439 & 1,609 & 2,196 & 2,035 & 2,358 & 49 & 46 & 52 \\
\hline World & 12,250 & 11,631 & 12,869 & 17,787 & 16,735 & 18,840 & 477 & 454 & 500 \\
\hline
\end{tabular}

having the greatest burden. The deaths and PYLL attributable to HIV/AIDS from not adhering to antiretroviral therapy by GBD region for 2004 per 10,000,000 people are outlined in Appendix Tables 2a and 2b. The Sub-Saharan Africa Southern, region had the highest number of HIV/AIDS-related deaths and PYLL per 10,000,000 people due to non-adherence to ART, with an estimated 3,530.92 (95\% CI: 3,311.90-3,749.94) deaths per 10,000,000 people and 81,949 (95\% CI: 76,948-86,949) PYLL per 10,000,000 people. In contrast, the Asia South region had the lowest number of HIV/AIDS-related deaths and PYLL per 10,000,000 people due to non-adherence to ART, with an estimated 1.95 (95\% CI: 1.71-2.18) deaths per 10,000,000 people and 50 (95\% CI: 44-56) PYLL per 10,000,000 people.
The prevalence of current abstainers and current drinkers designated by levels of alcohol consumption, age, and sex is outlined in Tables 3a and 3b. The prevalence of current drinkers and current abstainers varied greatly by region, age, and sex, with men having the largest prevalence of current drinkers in all regions. Overall, for both men and women, the age groups of 15 to 34 and of 35 to 64 years had the highest prevalence of current drinkers and heavy drinkers in all regions.

HIV/AIDS-related deaths due to non-adherence to ART because of alcohol consumption by GBD region, age, and sex are presented in Tables 4a and 4b. Overall, 8,000 (95\% CI: 3,000-13,000) HIV/AIDS deaths from non-adherence to ART were attributable to alcohol consumption. This can 
Table 2a

Potential years of life lost to HIV/AIDS from not adhering to antiretroviral therapy by Global Burden of Disease region for 2004 (Men)

\begin{tabular}{|c|c|c|c|c|c|c|c|c|c|}
\hline \multirow[b]{2}{*}{$\begin{array}{l}\text { Global Burden of } \\
\text { Disease region }\end{array}$} & \multicolumn{3}{|c|}{ 15-34 years } & \multicolumn{3}{|c|}{ 35-64 years } & \multicolumn{3}{|c|}{$65+$ years } \\
\hline & $\begin{array}{c}\begin{array}{c}\text { Point } \\
\text { estimate }\end{array} \\
\end{array}$ & $\begin{array}{c}\text { Lower } \\
\text { estimate }\end{array}$ & $\begin{array}{c}\text { Upper } \\
\text { estimate }\end{array}$ & $\begin{array}{c}\text { Point } \\
\text { estimate }\end{array}$ & $\begin{array}{c}\text { Lower } \\
\text { estimate }\end{array}$ & $\begin{array}{c}\text { Upper } \\
\text { estimate }\end{array}$ & $\begin{array}{c}\text { Point } \\
\text { estimate }\end{array}$ & $\begin{array}{c}\text { Lower } \\
\text { estimate }\end{array}$ & $\begin{array}{r}\text { Upper } \\
\text { estimate }\end{array}$ \\
\hline $\begin{array}{l}\text { Asia, Pacific } \\
\text { [High Income] }\end{array}$ & 340 & 296 & 384 & 619 & 541 & 697 & 23 & 21 & 26 \\
\hline Asia, Central & 178 & 158 & 197 & 131 & 113 & 148 & 1 & 0 & 1 \\
\hline Asia, East & 8,801 & 7,704 & 9,898 & 10,453 & 9,108 & 11,798 & 55 & 49 & 62 \\
\hline Asia, South & 14,813 & 14,184 & 15,441 & 9,262 & 8,704 & 9,820 & 36 & 35 & 38 \\
\hline Asia, Southeast & 49,621 & 46,110 & 53,132 & 49,548 & 46,576 & 52,519 & 256 & 243 & 270 \\
\hline Australasia & 92 & 80 & 104 & 250 & 217 & 284 & 13 & 12 & 15 \\
\hline Caribbean & 11,694 & 10,326 & 13,062 & 16,753 & 14,835 & 18,672 & 221 & 205 & 238 \\
\hline Europe, Central & 980 & 865 & 1,094 & 785 & 688 & 881 & 17 & 15 & 19 \\
\hline Europe, Eastern & 3,071 & 2,641 & 3,501 & 2,900 & 2,506 & 3,295 & 18 & 16 & 20 \\
\hline Europe, Western & 6,337 & 5,536 & 7,138 & 14,482 & 12,582 & 16,382 & 450 & 396 & 504 \\
\hline $\begin{array}{l}\text { Latin America, } \\
\text { Andean }\end{array}$ & 6,117 & 5,412 & 6,823 & 5,686 & 4,970 & 6,402 & 32 & 30 & 34 \\
\hline $\begin{array}{l}\text { Latin America, } \\
\text { Central }\end{array}$ & 30,686 & 27,757 & 33,614 & 30,210 & 26,910 & 33,510 & 352 & 322 & 383 \\
\hline $\begin{array}{l}\text { Latin America, } \\
\text { Southern }\end{array}$ & 4,335 & 3,777 & 4,892 & 6,371 & 5,586 & 7,156 & 144 & 128 & 160 \\
\hline $\begin{array}{l}\text { Latin America, } \\
\text { Tropical }\end{array}$ & 31,822 & 28,411 & 35,232 & 43,022 & 39,140 & 46,904 & 637 & 600 & 675 \\
\hline $\begin{array}{l}\text { Northern Africa / } \\
\text { Middle East }\end{array}$ & 1,523 & 1,481 & 1,564 & 1,242 & 1,195 & 1,289 & 5 & 5 & 6 \\
\hline $\begin{array}{l}\text { North America } \\
\text { [High Income] }\end{array}$ & 10,491 & 9,238 & 11,745 & 31,507 & 27,877 & 35,138 & 898 & 813 & 984 \\
\hline Oceania & 187 & 161 & 212 & 289 & 251 & 327 & 2 & 2 & 2 \\
\hline $\begin{array}{l}\text { Sub-Saharan } \\
\text { Africa, Central }\end{array}$ & 2,387 & 2,117 & 2,657 & 3,455 & 3,060 & 3,850 & 37 & 34 & 39 \\
\hline $\begin{array}{l}\text { Sub-Saharan } \\
\text { Africa, East }\end{array}$ & 54,459 & 51,073 & 57,846 & 92,700 & 84,781 & 100,619 & 1,416 & 1,299 & 1,534 \\
\hline $\begin{array}{l}\text { Sub-Saharan } \\
\text { Africa, Southern }\end{array}$ & 79,375 & 72,831 & 85,920 & 136,938 & 125,648 & 148,228 & 1,475 & 1,373 & 1,577 \\
\hline $\begin{array}{l}\text { Sub-Saharan } \\
\text { Africa, Western }\end{array}$ & 25,042 & 22,998 & 27,086 & 41,622 & 37,318 & 45,926 & 615 & 560 & 669 \\
\hline World & 342,350 & 313,157 & 371,543 & 498,226 & 452,606 & 543,847 & 6,705 & 6,158 & 7,253 \\
\hline
\end{tabular}

be broken down into 5,600 (95\% CI: 2,300-8,900) HIV/AIDS-related deaths in men and 2,400 (95\% CI: 8004,100) HIV/AIDS-related deaths in women from nonadherence to ART attributable to alcohol consumption. This represents $0.39 \%$ (95\% CI: $0.15 \%-0.64 \%)$ of all HIV/ AIDS-related deaths, $0.54 \%$ (95\%CI: $0.22 \%-0.86 \%$ ) of all HIV/AIDS-related deaths for men, and $0.24 \%$ (95\% CI: $0.07 \%-0.41 \%$ ) of all HIV/AIDS-related deaths for women. Of these totals, 2,646 (1,898 for men and 748 for women) HIV/AIDS-related deaths were attributable to heavy alcohol consumption. Thus an estimated $11.9 \%$ (15.1\% for men and $8.0 \%$ for women) of all HIV/AIDS-related deaths from non-adherence to ART were attributable to alcohol consumption and 3.93\% (5.15\% for men and $2.45 \%$ for women) of all HIV/AIDS-related deaths from nonadherence to ART were attributable to heavy alcohol consumption. The alcohol-attributable fractions for HIV from not adhering to ART because of alcohol consumption by GBD region for 2004 are outlined in Appendix Tables 3a and $3 \mathrm{~b}$. The deaths attributable to HIV/AIDS from not adhering to ART because of heavy alcohol consumption by GBD isease region for 2004 is outlined in Appendix Tables $4 \mathrm{a}$ and $4 \mathrm{~b}$.

The number of HIV/AIDS-related deaths per 10,000,000 people attributable to non-adherence to ART due to alcohol consumption and to heavy alcohol consumption are presented in Figures 1 and 2 respectively. The SubSaharan Africa, Southern, region had the highest rate of HIV/AIDS-related deaths from non-adherence to ART because of alcohol consumption, with 320.39 (95\% CI: 101.37-539.40) deaths per $10,000,000$ people, of which 
Table 2b

Potential years of life lost to HIV/AIDS from not adhering to antiretroviral therapy by Global Burden of Disease region for 2004 (Women)

\begin{tabular}{|c|c|c|c|c|c|c|c|c|c|}
\hline \multirow[b]{2}{*}{$\begin{array}{l}\text { Global Burden of } \\
\text { Disease region }\end{array}$} & \multicolumn{3}{|c|}{ 15-34 years } & \multicolumn{3}{|c|}{ 35-64 years } & \multicolumn{3}{|c|}{$65+$ years } \\
\hline & $\begin{array}{c}\text { Point } \\
\text { estimate }\end{array}$ & $\begin{array}{c}\text { Lower } \\
\text { estimate }\end{array}$ & $\begin{array}{c}\text { Upper } \\
\text { estimate }\end{array}$ & $\begin{array}{c}\begin{array}{c}\text { Point } \\
\text { estimate }\end{array} \\
\end{array}$ & $\begin{array}{c}\text { Lower } \\
\text { estimate }\end{array}$ & $\begin{array}{c}\text { Upper } \\
\text { estimate }\end{array}$ & $\begin{array}{c}\text { Point } \\
\text { estimate }\end{array}$ & $\begin{array}{c}\text { Lower } \\
\text { estimate }\end{array}$ & $\begin{array}{c}\text { Upper } \\
\text { estimate }\end{array}$ \\
\hline $\begin{array}{l}\text { Asia, Pacific } \\
\text { [High Income] }\end{array}$ & 101 & 88 & 114 & 84 & 74 & 94 & 3 & 3 & 3 \\
\hline Asia, Central & 39 & 36 & 42 & 24 & 21 & 27 & 0 & 0 & 0 \\
\hline Asia, East & 2,599 & 2,384 & 2,813 & 2,414 & 2,215 & 2,613 & 23 & 21 & 24 \\
\hline Asia, South & 5,458 & 5,376 & 5,540 & 2,487 & 2,443 & 2,532 & 24 & 24 & 24 \\
\hline Asia, Southeast & 16,116 & 15,676 & 16,556 & 12,685 & 12,422 & 12,949 & 144 & 142 & 146 \\
\hline Australasia & 22 & 19 & 25 & 25 & 22 & 28 & 1 & 1 & 2 \\
\hline Caribbean & 8,195 & 7,452 & 8,938 & 8,130 & 7,616 & 8,643 & 106 & 102 & 109 \\
\hline Europe, Central & 619 & 547 & 690 & 264 & 235 & 292 & 0 & 0 & 0 \\
\hline Europe, Eastern & 885 & 782 & 987 & 679 & 602 & 755 & 12 & 11 & 13 \\
\hline Europe, Western & 2,192 & 1,920 & 2,464 & 3,776 & 3,302 & 4,251 & 104 & 95 & 114 \\
\hline $\begin{array}{l}\text { Latin America, } \\
\text { Andean }\end{array}$ & 1,903 & 1,727 & 2,079 & 1,399 & 1,267 & 1,531 & 16 & 15 & 17 \\
\hline $\begin{array}{l}\text { Latin America, } \\
\text { Central }\end{array}$ & 9,692 & 8,971 & 10,412 & 7,479 & 6,945 & 8,014 & 99 & 93 & 105 \\
\hline $\begin{array}{l}\text { Latin America, } \\
\text { Southern }\end{array}$ & 1,581 & 1,397 & 1,765 & 1,674 & 1,505 & 1,842 & 34 & 30 & 37 \\
\hline $\begin{array}{l}\text { Latin America, } \\
\text { Tropical }\end{array}$ & 18,153 & 16,558 & 19,749 & 21,861 & 20,298 & 23,424 & 379 & 361 & 397 \\
\hline $\begin{array}{l}\text { Northern Africa / } \\
\text { Middle East }\end{array}$ & 446 & 438 & 454 & 292 & 289 & 294 & 3 & 3 & 3 \\
\hline $\begin{array}{l}\text { North America } \\
\text { [High Income] }\end{array}$ & 5,235 & 4,646 & 5,824 & 11,813 & 10,561 & 13,064 & 278 & 260 & 297 \\
\hline Oceania & 110 & 101 & 119 & 110 & 100 & 121 & 1 & 1 & 1 \\
\hline $\begin{array}{l}\text { Sub-Saharan } \\
\text { Africa, Central }\end{array}$ & 4,529 & 4,160 & 4,899 & 4,660 & 4,314 & 5,006 & 52 & 49 & 54 \\
\hline $\begin{array}{l}\text { Sub-Saharan } \\
\text { Africa, East }\end{array}$ & 104,754 & 99,889 & 109,619 & 121,551 & 113,937 & 129,165 & 1,062 & 1,002 & 1,122 \\
\hline $\begin{array}{l}\text { Sub-Saharan } \\
\text { Africa, Southern }\end{array}$ & 129,312 & 124,259 & 134,365 & 149,326 & 142,597 & 156,055 & 1,493 & 1,443 & 1,542 \\
\hline $\begin{array}{l}\text { Sub-Saharan } \\
\text { Africa, Western }\end{array}$ & 44,327 & 41,866 & 46,788 & 49,574 & 45,927 & 53,221 & 467 & 436 & 498 \\
\hline World & 356,268 & 338,294 & 374,243 & 400,307 & 376,691 & 423,923 & 4,298 & 4,091 & 4,505 \\
\hline
\end{tabular}

154.54 (95\% CI: 59.46-249.63) were caused by heavy alcohol consumption. The Northern Africa/Middle East region had the lowest rate of HIV/AIDS-related deaths from non-adherence to ART because of alcohol consumption, with 0.11 (95\% CI: 0.01-0.22) deaths per 10,000,000 people, of which 0.03 (95\% CI: 0.01-0.05) were attributable to heavy alcohol consumption.

PYLL caused by non-adherence to ART because of alcohol consumption for both men and women by GBD region are outlined in Tables 5a and 5b. In 2004 there were an estimated 187,000 (95\% CI: 70,000-304,000) PYLL from not adhering to ART because of alcohol consumption. This indicates that $0.36 \%$ (95\% CI: $0.13 \%-0.58 \%)$ of all PYLL are caused by non-adherence to ART due to alcohol consumption. This can be broken down into 127,000 (95\%
CI: 51,000-202,000) PYLL, representing 0.31\% (95\% CI: $0.03 \%-0.69 \%$ ) of all PYLL caused by HIV/AIDS for men, and $60,000 \quad(95 \%$ CI: 18,000 to 102,000$)$ PYLL, representing $0.22 \%$ (95\% CI: $0.07 \%-0.38 \%$ ) of all PYLL caused by HIV/AIDS for women. Thus, an estimated $11.6 \%$ (15.0\% for men and $7.9 \%$ for women) of all HIV/AIDS-related PYLL from non-adherence to ART were attributable to alcohol consumption and 3.80\% (5.06\% for men and 2.41\% for women) of all HIV/AIDS-related PYLL from non-adherence to ART were attributable to heavy alcohol consumption. The PYLL attributable to HIV/AIDS from not adhering to ART because of heavy alcohol consumption by GBD region for 2004 is outlined in Appendix Tables 5a and 5b. 
Table 3a

Drinking indicators by Global Burden of Disease region for 2005 (Men)

\begin{tabular}{|c|c|c|c|c|c|c|c|c|c|}
\hline \multirow[b]{2}{*}{$\begin{array}{l}\text { Global Burden of } \\
\text { Disease region }\end{array}$} & \multicolumn{3}{|c|}{ 15-34 years } & \multicolumn{3}{|c|}{ 35-64 years } & \multicolumn{3}{|c|}{$65+$ years } \\
\hline & \multicolumn{2}{|c|}{$\begin{array}{l}\text { Current drinkers } \\
0 \text { to }\end{array}$} & $\begin{array}{c}\text { Current } \\
\text { abstainers }\end{array}$ & \multicolumn{2}{|c|}{$\begin{array}{l}\text { Current drinkers } \\
0 \text { to }\end{array}$} & $\begin{array}{c}\text { Current } \\
\text { abstainers }\end{array}$ & \multicolumn{2}{|c|}{$\begin{array}{l}\text { Current drinkers } \\
0 \text { to }\end{array}$} & $\begin{array}{c}\text { Current } \\
\text { abstainers }\end{array}$ \\
\hline $\begin{array}{l}\text { Asia, Pacific } \\
\text { [High Income] }\end{array}$ & $84.9 \%$ & $8.0 \%$ & $7.2 \%$ & $73.4 \%$ & $16.2 \%$ & $10.4 \%$ & $64.4 \%$ & $6.1 \%$ & $29.5 \%$ \\
\hline Asia, Central & $48.0 \%$ & $8.1 \%$ & $43.9 \%$ & $67.5 \%$ & $9.1 \%$ & $23.4 \%$ & $49.0 \%$ & $4.0 \%$ & $47.0 \%$ \\
\hline Asia, East & $69.5 \%$ & $1.1 \%$ & $29.4 \%$ & $62.4 \%$ & $11.7 \%$ & $25.9 \%$ & $57.7 \%$ & $6.5 \%$ & $35.8 \%$ \\
\hline Asia, South & $11.1 \%$ & $2.1 \%$ & $86.8 \%$ & $17.2 \%$ & $4.8 \%$ & $78.1 \%$ & $10.4 \%$ & $2.5 \%$ & $87.0 \%$ \\
\hline Asia, Southeast & $28.5 \%$ & $2.2 \%$ & $69.3 \%$ & $18.1 \%$ & $6.0 \%$ & $75.9 \%$ & $17.0 \%$ & $2.9 \%$ & $80.1 \%$ \\
\hline Australasia & $78.0 \%$ & $9.4 \%$ & $12.6 \%$ & $77.1 \%$ & $12.9 \%$ & $10.1 \%$ & $68.5 \%$ & $8.2 \%$ & $23.3 \%$ \\
\hline Caribbean & $63.8 \%$ & $5.8 \%$ & $30.4 \%$ & $59.3 \%$ & $8.0 \%$ & $32.6 \%$ & $32.0 \%$ & $3.6 \%$ & $64.4 \%$ \\
\hline Europe, Central & $60.1 \%$ & $14.7 \%$ & $25.2 \%$ & $60.2 \%$ & $21.4 \%$ & $18.4 \%$ & $54.8 \%$ & $15.4 \%$ & $29.8 \%$ \\
\hline Europe, Eastern & $53.4 \%$ & $21.7 \%$ & $24.8 \%$ & $52.1 \%$ & $19.7 \%$ & $28.1 \%$ & $46.6 \%$ & $13.1 \%$ & $40.3 \%$ \\
\hline Europe, Western & $73.5 \%$ & $12.8 \%$ & $13.6 \%$ & $74.8 \%$ & $17.1 \%$ & $8.2 \%$ & $68.9 \%$ & $10.0 \%$ & $21.1 \%$ \\
\hline $\begin{array}{l}\text { Latin America, } \\
\text { Andean }\end{array}$ & $60.3 \%$ & $7.2 \%$ & $32.6 \%$ & $65.2 \%$ & $12.2 \%$ & $22.6 \%$ & $21.1 \%$ & $1.1 \%$ & $77.8 \%$ \\
\hline $\begin{array}{l}\text { Latin America, } \\
\text { Central }\end{array}$ & $44.8 \%$ & $8.6 \%$ & $46.6 \%$ & $54.1 \%$ & $11.6 \%$ & $34.2 \%$ & $38.2 \%$ & $7.3 \%$ & $54.5 \%$ \\
\hline $\begin{array}{l}\text { Latin America, } \\
\text { Southern }\end{array}$ & $81.0 \%$ & $10.7 \%$ & $8.3 \%$ & $74.2 \%$ & $11.1 \%$ & $14.7 \%$ & $62.4 \%$ & $7.2 \%$ & $30.4 \%$ \\
\hline $\begin{array}{l}\text { Latin America, } \\
\text { Tropical }\end{array}$ & $55.2 \%$ & $13.6 \%$ & $31.2 \%$ & $40.8 \%$ & $11.5 \%$ & $47.7 \%$ & $24.5 \%$ & $2.6 \%$ & $72.9 \%$ \\
\hline $\begin{array}{l}\text { Northern Africa / } \\
\text { Middle East }\end{array}$ & $5.3 \%$ & $1.9 \%$ & $92.8 \%$ & $10.5 \%$ & $1.5 \%$ & $88.0 \%$ & $5.5 \%$ & $0.0 \%$ & $94.5 \%$ \\
\hline $\begin{array}{l}\text { North America } \\
\text { [High Income] }\end{array}$ & $62.6 \%$ & $15.4 \%$ & $21.9 \%$ & $62.9 \%$ & $10.8 \%$ & $26.3 \%$ & $49.5 \%$ & $5.1 \%$ & $45.5 \%$ \\
\hline Oceania & $81.2 \%$ & $0.9 \%$ & $17.9 \%$ & $75.5 \%$ & $1.7 \%$ & $22.8 \%$ & $68.5 \%$ & $0.3 \%$ & $31.2 \%$ \\
\hline $\begin{array}{l}\text { Sub-Saharan } \\
\text { Africa, Central }\end{array}$ & $47.9 \%$ & $3.1 \%$ & $49.0 \%$ & $47.9 \%$ & $4.0 \%$ & $48.1 \%$ & $19.9 \%$ & $0.6 \%$ & $79.5 \%$ \\
\hline $\begin{array}{l}\text { Sub-Saharan } \\
\text { Africa, East }\end{array}$ & $20.3 \%$ & $4.3 \%$ & $75.4 \%$ & $29.1 \%$ & $9.7 \%$ & $61.2 \%$ & $31.9 \%$ & $5.3 \%$ & $62.8 \%$ \\
\hline $\begin{array}{l}\text { Sub-Saharan } \\
\text { Africa, Southern }\end{array}$ & $26.8 \%$ & $11.2 \%$ & $62.0 \%$ & $26.8 \%$ & $11.2 \%$ & $62.0 \%$ & $24.8 \%$ & $4.7 \%$ & $70.5 \%$ \\
\hline $\begin{array}{l}\text { Sub-Saharan } \\
\text { Africa, Western }\end{array}$ & $29.6 \%$ & $6.6 \%$ & $63.8 \%$ & $38.9 \%$ & $12.0 \%$ & $49.1 \%$ & $36.6 \%$ & $3.9 \%$ & $59.5 \%$ \\
\hline World & $39.8 \%$ & $5.1 \%$ & $55.0 \%$ & $45.4 \%$ & $10.2 \%$ & $44.4 \%$ & $43.3 \%$ & $6.1 \%$ & $50.7 \%$ \\
\hline
\end{tabular}

The number of PYLL per 10,000,000 people for alcohol consumption and heavy alcohol consumption are presented in Figures 3 and 4 respectively. The Sub-Saharan Africa, Southern, region had the highest rate of HIV/AIDS-related PYLL from non-adherence to ART because of alcohol consumption, with 7,279 (95\% CI: 2,279-12,279) PYLL per 10,000,000 people, of which 3,542 (95\% CI: 1,364 5,721) were attributable to heavy alcohol consumption. The Northern Africa/Middle East region had the lowest rate of HIV/AIDS-related PYLL from non-adherence to ART because of alcohol consumption, with 3 (95\% CI: 0-6) PYLL per 10,000,000 people, of which 1 (95\% CI: 0-1) was attributable to heavy alcohol consumption.

\section{Discussion}

Previous evidence has shown that alcohol consumption has a large impact on adherence to ART; however, due to methodological limitations the overall mortality caused by not adhering to ART and the mortality caused by not adhering to ART because of alcohol consumption have previously not been quantified. Our findings indicate that this burden of mortality is non-trivial, and additional research is required concerning the effectiveness of different interventions aimed at reducing alcohol consumption among people with HIV/AIDS. These interventions may also have beneficial effects on other diseases to which 
Table 3b

Drinking indicators by Global Burden of Disease region for 2005 (Women)

\begin{tabular}{|c|c|c|c|c|c|c|c|c|c|}
\hline \multirow[b]{2}{*}{$\begin{array}{l}\text { Global Burden of } \\
\text { Disease region }\end{array}$} & \multicolumn{3}{|c|}{ 15-34 years } & \multicolumn{3}{|c|}{ 35-64 years } & \multicolumn{3}{|c|}{$65+$ years } \\
\hline & Current drinkers & $\begin{array}{l}\text { rinkers } \\
\qquad \begin{array}{c}40+ \\
\text { grams }\end{array}\end{array}$ & $\begin{array}{c}\text { Current } \\
\text { abstainers }\end{array}$ & $\begin{array}{l}\text { Current } \\
0 \text { to } \\
<40 \\
\text { grams }\end{array}$ & $\begin{array}{l}\text { Irinkers } \\
\begin{array}{c}\mathbf{4 0 +} \\
\text { grams }\end{array}\end{array}$ & $\begin{array}{l}\text { Current } \\
\text { abstainers }\end{array}$ & $\begin{array}{c}\text { Curren } \\
0 \text { to } \\
<40 \\
\text { grams }\end{array}$ & $\begin{array}{l}\text { |rinkers } \\
\begin{array}{l}\mathbf{4 0 +} \\
\text { grams }\end{array}\end{array}$ & $\begin{array}{c}\text { Current } \\
\text { abstainers }\end{array}$ \\
\hline $\begin{array}{l}\text { Asia, Pacific } \\
\text { [High Income] }\end{array}$ & $85.8 \%$ & $2.4 \%$ & $11.8 \%$ & $74.2 \%$ & $5.4 \%$ & $20.5 \%$ & $49.8 \%$ & $0.4 \%$ & $49.8 \%$ \\
\hline Asia, Central & $32.7 \%$ & $1.8 \%$ & $65.4 \%$ & $61.0 \%$ & $4.4 \%$ & $34.5 \%$ & $32.9 \%$ & $0.3 \%$ & $66.8 \%$ \\
\hline Asia, East & $38.3 \%$ & $0.0 \%$ & $61.7 \%$ & $35.6 \%$ & $2.8 \%$ & $61.7 \%$ & $29.9 \%$ & $0.7 \%$ & $69.5 \%$ \\
\hline Asia, South & $2.4 \%$ & $0.1 \%$ & $97.6 \%$ & $2.7 \%$ & $0.6 \%$ & $96.7 \%$ & $0.4 \%$ & $0.0 \%$ & $99.6 \%$ \\
\hline Asia, Southeast & $6.9 \%$ & $0.3 \%$ & $92.8 \%$ & $3.8 \%$ & $0.8 \%$ & $95.5 \%$ & $1.9 \%$ & $0.0 \%$ & $98.1 \%$ \\
\hline Australasia & $77.4 \%$ & $5.6 \%$ & $17.0 \%$ & $80.5 \%$ & $5.8 \%$ & $13.7 \%$ & $54.7 \%$ & $2.2 \%$ & $43.1 \%$ \\
\hline Caribbean & $42.0 \%$ & $5.2 \%$ & $52.8 \%$ & $26.7 \%$ & $1.0 \%$ & $72.4 \%$ & $8.7 \%$ & $0.1 \%$ & $91.3 \%$ \\
\hline Europe, Central & $63.0 \%$ & $10.2 \%$ & $26.8 \%$ & $55.4 \%$ & $10.7 \%$ & $33.9 \%$ & $16.9 \%$ & $0.8 \%$ & $82.4 \%$ \\
\hline Europe, Eastern & $42.4 \%$ & $14.4 \%$ & $43.2 \%$ & $42.5 \%$ & $12.5 \%$ & $45.0 \%$ & $29.3 \%$ & $2.5 \%$ & $68.1 \%$ \\
\hline Europe, Western & $78.3 \%$ & $5.6 \%$ & $16.1 \%$ & $74.3 \%$ & $11.2 \%$ & $14.5 \%$ & $49.8 \%$ & $3.6 \%$ & $46.6 \%$ \\
\hline $\begin{array}{l}\text { Latin America, } \\
\text { Andean }\end{array}$ & $44.3 \%$ & $4.0 \%$ & $51.6 \%$ & $47.8 \%$ & $2.1 \%$ & $50.1 \%$ & $26.4 \%$ & $1.2 \%$ & $72.4 \%$ \\
\hline $\begin{array}{l}\text { Latin America, } \\
\text { Central }\end{array}$ & $31.8 \%$ & $4.7 \%$ & $63.4 \%$ & $31.5 \%$ & $3.0 \%$ & $65.5 \%$ & $26.3 \%$ & $0.3 \%$ & $73.4 \%$ \\
\hline $\begin{array}{l}\text { Latin America, } \\
\text { Southern }\end{array}$ & $74.1 \%$ & $3.4 \%$ & $22.5 \%$ & $53.8 \%$ & $7.9 \%$ & $38.3 \%$ & $49.3 \%$ & $2.3 \%$ & $48.4 \%$ \\
\hline $\begin{array}{l}\text { Latin America, } \\
\text { Tropical }\end{array}$ & $45.0 \%$ & $5.2 \%$ & $49.8 \%$ & $29.9 \%$ & $6.6 \%$ & $63.5 \%$ & $18.6 \%$ & $0.5 \%$ & $80.8 \%$ \\
\hline $\begin{array}{l}\text { Northern Africa / } \\
\text { Middle East }\end{array}$ & $3.1 \%$ & $0.5 \%$ & $96.5 \%$ & $0.9 \%$ & $0.2 \%$ & $98.9 \%$ & $0.3 \%$ & $0.0 \%$ & $99.6 \%$ \\
\hline $\begin{array}{l}\text { North America } \\
\text { [High Income] }\end{array}$ & $63.7 \%$ & $7.2 \%$ & $29.0 \%$ & $59.2 \%$ & $5.3 \%$ & $35.6 \%$ & $31.2 \%$ & $1.0 \%$ & $67.8 \%$ \\
\hline Oceania & $47.6 \%$ & $0.0 \%$ & $52.4 \%$ & $48.0 \%$ & $0.1 \%$ & $52.0 \%$ & $43.5 \%$ & $0.0 \%$ & $56.5 \%$ \\
\hline $\begin{array}{l}\text { Sub-Saharan } \\
\text { Africa, Central }\end{array}$ & $30.6 \%$ & $1.8 \%$ & $67.6 \%$ & $25.9 \%$ & $2.5 \%$ & $71.5 \%$ & $11.6 \%$ & $0.3 \%$ & $88.1 \%$ \\
\hline $\begin{array}{l}\text { Sub-Saharan } \\
\text { Africa, East }\end{array}$ & $14.1 \%$ & $1.9 \%$ & $83.9 \%$ & $20.3 \%$ & $4.5 \%$ & $75.1 \%$ & $19.7 \%$ & $1.7 \%$ & $78.6 \%$ \\
\hline $\begin{array}{l}\text { Sub-Saharan } \\
\text { Africa, Southern }\end{array}$ & $8.5 \%$ & $4.1 \%$ & $87.4 \%$ & $11.3 \%$ & $4.4 \%$ & $84.3 \%$ & $8.5 \%$ & $1.2 \%$ & $90.2 \%$ \\
\hline $\begin{array}{l}\text { Sub-Saharan } \\
\text { Africa, Western }\end{array}$ & $17.6 \%$ & $3.2 \%$ & $79.2 \%$ & $24.0 \%$ & $7.2 \%$ & $68.8 \%$ & $24.8 \%$ & $2.3 \%$ & $72.9 \%$ \\
\hline World & $26.5 \%$ & $2.1 \%$ & $71.3 \%$ & $31.1 \%$ & $4.0 \%$ & $64.9 \%$ & $26.6 \%$ & $1.2 \%$ & $72.2 \%$ \\
\hline
\end{tabular}

alcohol consumption is causally related (Rehm et al., 2010).

There are limitations in the methodology used to estimate the HIV/AIDS-related mortality from non-adherence to ART due to alcohol consumption. The very broad categories used in this method for alcohol consumption and adherence to ART do not allow us to take into account different types of drinking patterns (e.g., moderate versus heavy drinking patterns) or to account for different percentages of non-adherence. This is especially important for regions with high variations in drinking patterns and for comparisons across regions. For example, in Africa many people abstain from alcohol, but those who do drink tend to do so heavily (WHO, 2011). Since we expect heavier drinking patterns to have a greater impact on ART adherence, and adherence to medication regimes to have a continuous effect on the rate of HIV/AIDS-related mortality (Hendershot et al., 2009; Marcellin et al., 2008), future research will be necessary to obtain more accurate estimates of the burden of HIV/AIDS-related mortality caused by non-adherence to ART because of alcohol consumption.

There are also limitations in our alcohol exposure and mortality data. The alcohol exposure data used to calculate the estimates presented in this study came from large population surveys, which have limitations with respect to 
Table 4a

Deaths attributable to HIV/AIDS from not adhering to antiretroviral therapy because of alcohol consumption by Global Burden of Disease region for 2004 (Men)

\begin{tabular}{|c|c|c|c|c|c|c|c|c|c|}
\hline \multirow[b]{2}{*}{$\begin{array}{l}\text { Global Burden of } \\
\text { Disease region }\end{array}$} & \multicolumn{3}{|c|}{ 15-34 years } & \multicolumn{3}{|c|}{ 35-64 years } & \multicolumn{3}{|c|}{$65+$ years } \\
\hline & $\begin{array}{c}\text { Point } \\
\text { estimate }\end{array}$ & $\begin{array}{c}\text { Lower } \\
\text { estimate }\end{array}$ & $\begin{array}{c}\text { Upper } \\
\text { estimate }\end{array}$ & $\begin{array}{c}\text { Point } \\
\text { estimate }\end{array}$ & $\begin{array}{c}\text { Lower } \\
\text { estimate }\end{array}$ & $\begin{array}{c}\text { Upper } \\
\text { estimate }\end{array}$ & $\begin{array}{c}\text { Point } \\
\text { estimate }\end{array}$ & $\begin{array}{l}\text { Lower } \\
\text { estimate }\end{array}$ & $\begin{array}{c}\text { Upper } \\
\text { estimate }\end{array}$ \\
\hline $\begin{array}{l}\text { Asia, Pacific } \\
\text { [High Income] }\end{array}$ & 3 & 2 & 5 & 8 & 4 & 12 & 1 & 0 & 1 \\
\hline Asia, Central & 1 & 0 & 2 & 1 & 1 & 2 & 0 & 0 & 0 \\
\hline Asia, East & 66 & 28 & 104 & 110 & 47 & 173 & 1 & 1 & 2 \\
\hline Asia, South & 24 & 3 & 45 & 33 & 8 & 58 & 0 & 0 & 0 \\
\hline Asia, Southeast & 181 & 62 & 301 & 201 & 62 & 340 & 2 & 1 & 4 \\
\hline Australasia & 1 & 0 & 1 & 3 & 2 & 5 & 0 & 0 & 1 \\
\hline Caribbean & 89 & 41 & 138 & 168 & 76 & 259 & 3 & 1 & 5 \\
\hline Europe, Central & 7 & 3 & 11 & 9 & 4 & 13 & 1 & 0 & 1 \\
\hline Europe, Eastern & 23 & 9 & 38 & 30 & 12 & 49 & 0 & 0 & 1 \\
\hline Europe, Western & 63 & 31 & 94 & 186 & 94 & 278 & 15 & 8 & 23 \\
\hline $\begin{array}{l}\text { Latin America, } \\
\text { Andean }\end{array}$ & 43 & 19 & 67 & 62 & 28 & 95 & 0 & 0 & 1 \\
\hline $\begin{array}{l}\text { Latin America, } \\
\text { Central }\end{array}$ & 181 & 81 & 280 & 290 & 136 & 444 & 7 & 3 & 11 \\
\hline $\begin{array}{l}\text { Latin America, } \\
\text { Southern }\end{array}$ & 41 & 21 & 61 & 75 & 38 & 112 & 4 & 2 & 6 \\
\hline $\begin{array}{l}\text { Latin America, } \\
\text { Tropical }\end{array}$ & 240 & 119 & 361 & 343 & 162 & 525 & 8 & 3 & 13 \\
\hline $\begin{array}{l}\text { Northern Africa / } \\
\text { Middle East }\end{array}$ & 1 & 0 & 3 & 3 & 0 & 5 & 0 & 0 & 0 \\
\hline $\begin{array}{l}\text { North America } \\
\text { [High Income] }\end{array}$ & 96 & 47 & 145 & 372 & 181 & 563 & 21 & 9 & 32 \\
\hline Oceania & 2 & 1 & 3 & 3 & 1 & 5 & 0 & 0 & 0 \\
\hline $\begin{array}{l}\text { Sub-Saharan } \\
\text { Africa, Central }\end{array}$ & 14 & 5 & 24 & 28 & 9 & 47 & 0 & 0 & 1 \\
\hline $\begin{array}{l}\text { Sub-Saharan } \\
\text { Africa, East }\end{array}$ & 174 & 51 & 297 & 594 & 210 & 977 & 21 & 7 & 34 \\
\hline $\begin{array}{l}\text { Sub-Saharan } \\
\text { Africa, Southern }\end{array}$ & 378 & 138 & 618 & 851 & 310 & 1,391 & 17 & 6 & 28 \\
\hline $\begin{array}{l}\text { Sub-Saharan } \\
\text { Africa, Western }\end{array}$ & 112 & 39 & 186 & 336 & 128 & 545 & 9 & 3 & 15 \\
\hline World & 1,742 & 702 & 2,782 & 3,705 & 1,513 & 5,898 & 111 & 45 & 178 \\
\hline
\end{tabular}

population coverage (Shield \& Rehm, 2012). The survey instruments commonly have inherent biases, leading to underestimation of the prevalence of current drinkers which, in turn, leads to an underestimation of the HIV/AIDS mortality caused by non-adherence to ART because of alcohol consumption (Shield \& Rehm, 2012). In addition, the mortality data used in our study are limited and have variations (Lopez et al., 2006b). For example, some countries, such as Ethiopia, do not have available information on numerous causes of death. In these instances, data on the missing causes of death are provided by WHO, which statistically estimates the number of deaths from these causes using information such as mortality profiles of other countries that are in the same geographic region (Lopez et al., 2006b).
Our main analysis is also limited by the fact that it does not include people younger than 15 years of age, because they tend not to consume alcohol. Although this is not a problem for the estimates of the alcohol-attributable burden of HIV/AIDS-related mortality, there may be people younger than 15 years who take ART. If our analysis is extended to include individuals of all ages, we estimate that 76,500 (95\% CI: 71,100-81,800) deaths and 1,922,000 (95\% CI: 1,791,000-2,054,000) PYLL were attributable to non-adherence to ART.

Although the association between alcohol consumption and adherence to ART is replicable and reliable, there is a lack of research on the interpersonal and situational moderators of the effect of alcohol consumption on adherence to ART, 
Table 4b

Deaths attributable to HIV/AIDS from not adhering to antiretroviral therapy because of alcohol consumption by Global Burden of Disease region for 2004 (Women)

\begin{tabular}{|c|c|c|c|c|c|c|c|c|c|}
\hline \multirow[b]{2}{*}{$\begin{array}{l}\text { Global Burden of } \\
\text { Disease region }\end{array}$} & \multicolumn{3}{|c|}{ 15-34 years } & \multicolumn{3}{|c|}{ 35-64 years } & \multicolumn{3}{|c|}{$65+$ years } \\
\hline & $\begin{array}{c}\text { Point } \\
\text { estimate }\end{array}$ & $\begin{array}{c}\text { Lower } \\
\text { estimate }\end{array}$ & $\begin{array}{c}\text { Upper } \\
\text { estimate }\end{array}$ & $\begin{array}{c}\text { Point } \\
\text { estimate }\end{array}$ & $\begin{array}{c}\text { Lower } \\
\text { estimate }\end{array}$ & $\begin{array}{c}\text { Upper } \\
\text { estimate }\end{array}$ & $\begin{array}{c}\text { Point } \\
\text { estimate }\end{array}$ & $\begin{array}{c}\text { Lower } \\
\text { estimate }\end{array}$ & $\begin{array}{c}\text { Upper } \\
\text { estimate }\end{array}$ \\
\hline $\begin{array}{l}\text { Asia, Pacific } \\
\text { [High Income] }\end{array}$ & 1 & 0 & 1 & 1 & 0 & 1 & 0 & 0 & 0 \\
\hline Asia, Central & 0 & 0 & 0 & 0 & 0 & 0 & 0 & 0 & 0 \\
\hline Asia, East & 11 & 4 & 19 & 14 & 5 & 23 & 0 & 0 & 0 \\
\hline Asia, South & 2 & 0 & 4 & 1 & 0 & 3 & 0 & 0 & 0 \\
\hline Asia, Southeast & 15 & 0 & 29 & 10 & 0 & 22 & 0 & 0 & 0 \\
\hline Australasia & 0 & 0 & 0 & 0 & 0 & 0 & 0 & 0 & 0 \\
\hline Caribbean & 44 & 18 & 69 & 35 & 12 & 57 & 0 & 0 & 1 \\
\hline Europe, Central & 4 & 2 & 6 & 2 & 1 & 4 & 0 & 0 & 0 \\
\hline Europe, Eastern & 5 & 2 & 9 & 6 & 2 & 9 & 0 & 0 & 0 \\
\hline Europe, Western & 20 & 10 & 30 & 43 & 21 & 64 & 2 & 1 & 4 \\
\hline $\begin{array}{l}\text { Latin America, } \\
\text { Andean }\end{array}$ & 10 & 4 & 16 & 10 & 4 & 16 & 0 & 0 & 0 \\
\hline $\begin{array}{l}\text { Latin America, } \\
\text { Central }\end{array}$ & 40 & 16 & 64 & 40 & 16 & 63 & 1 & 0 & 2 \\
\hline $\begin{array}{l}\text { Latin America, } \\
\text { Southern }\end{array}$ & 13 & 6 & 19 & 15 & 7 & 22 & 1 & 0 & 1 \\
\hline $\begin{array}{l}\text { Latin America, } \\
\text { Tropical }\end{array}$ & 103 & 48 & 158 & 125 & 54 & 196 & 3 & 1 & 6 \\
\hline $\begin{array}{l}\text { Northern Africa / } \\
\text { Middle East }\end{array}$ & 0 & 0 & 0 & 0 & 0 & 0 & 0 & 0 & 0 \\
\hline $\begin{array}{l}\text { North America } \\
\text { [High Income] }\end{array}$ & 42 & 20 & 64 & 117 & 56 & 178 & 4 & 2 & 7 \\
\hline Oceania & 0 & 0 & 1 & 1 & 0 & 1 & 0 & 0 & 0 \\
\hline $\begin{array}{l}\text { Sub-Saharan } \\
\text { Africa, Central }\end{array}$ & 17 & 5 & 30 & 21 & 5 & 36 & 0 & 0 & 0 \\
\hline $\begin{array}{l}\text { Sub-Saharan } \\
\text { Africa, East }\end{array}$ & 215 & 47 & 383 & 478 & 141 & 814 & 9 & 2 & 15 \\
\hline $\begin{array}{l}\text { Sub-Saharan } \\
\text { Africa, Southern }\end{array}$ & 212 & 37 & 387 & 383 & 86 & 681 & 6 & 1 & 11 \\
\hline $\begin{array}{l}\text { Sub-Saharan } \\
\text { Africa, Western }\end{array}$ & 115 & 31 & 200 & 240 & 78 & 401 & 5 & 1 & 8 \\
\hline World & 870 & 252 & 1,489 & 1,541 & 491 & 2,594 & 33 & 9 & 56 \\
\hline
\end{tabular}

with alcohol consumption decreasing cognitive capacity, thereby decreasing the ability to remember to take medication and impairing judgment (Braithwaite et al., 2008; Hendershot et al., 2009; Parsons, Rosof, \& Mustanski, 2008). In addition the magnitude of the effect of alcohol on adherence to ART may be affected by alcohol users having misconceptions of the possible detrimental interactions between alcohol and medications (Brigido et al., 2001; Kalichman et al., 2009; Sankar, Wunderlich, Neufeld, \& Luborsky, 2007), and the use of alcohol to reduce the impact of the negative effects of HIV/AIDS (McKirnan, Ostrow, \& Hope, 1996; Nemeroff, Hoyt, Huebner, \& Proescholdbell, 2008). Despite the limitations in methodology used to estimate the burden of HIV/AIDS due to non-adherence to ART attributable to alcohol consumption, the finding is still valid that alcohol has a large impact on HIV/AIDS mortality due to people not adhering to ART.

\section{Conclusions}

Our results demonstrate that in 2004 alcohol was attributable for 8,000 (95\% CI: 3,000-13,000) HIV/AIDSrelated deaths and 187,000 (95\% CI: 70,000-304,000) HIV/AIDS-related PYLL caused by non-adherence to ART because of alcohol consumption. This represents a portion of the HIV/AIDS-related deaths and PYLL that are attributable to alcohol. Additional research is needed to quantify the HIV/AIDS-related deaths caused by unsafe sex and by the worsening of the disease course other than by non-adherence to ART, such as a weakening of the immune system (Braithwaite et al., 2005; Hahn \& Samet, 2010; Neuman et al., 2006). Our research indicates that alcohol has a non-trivial impact on mortality for both men and women from HIV/AIDS-related deaths due to nonadherence to ART. 


\section{Figure 1}

Deaths attributable to HIV/AIDS from not adhering to antiretroviral therapy because of alcohol consumption by Global Burden of Disease region for 2004 per 10,000,000 people 15 years or older

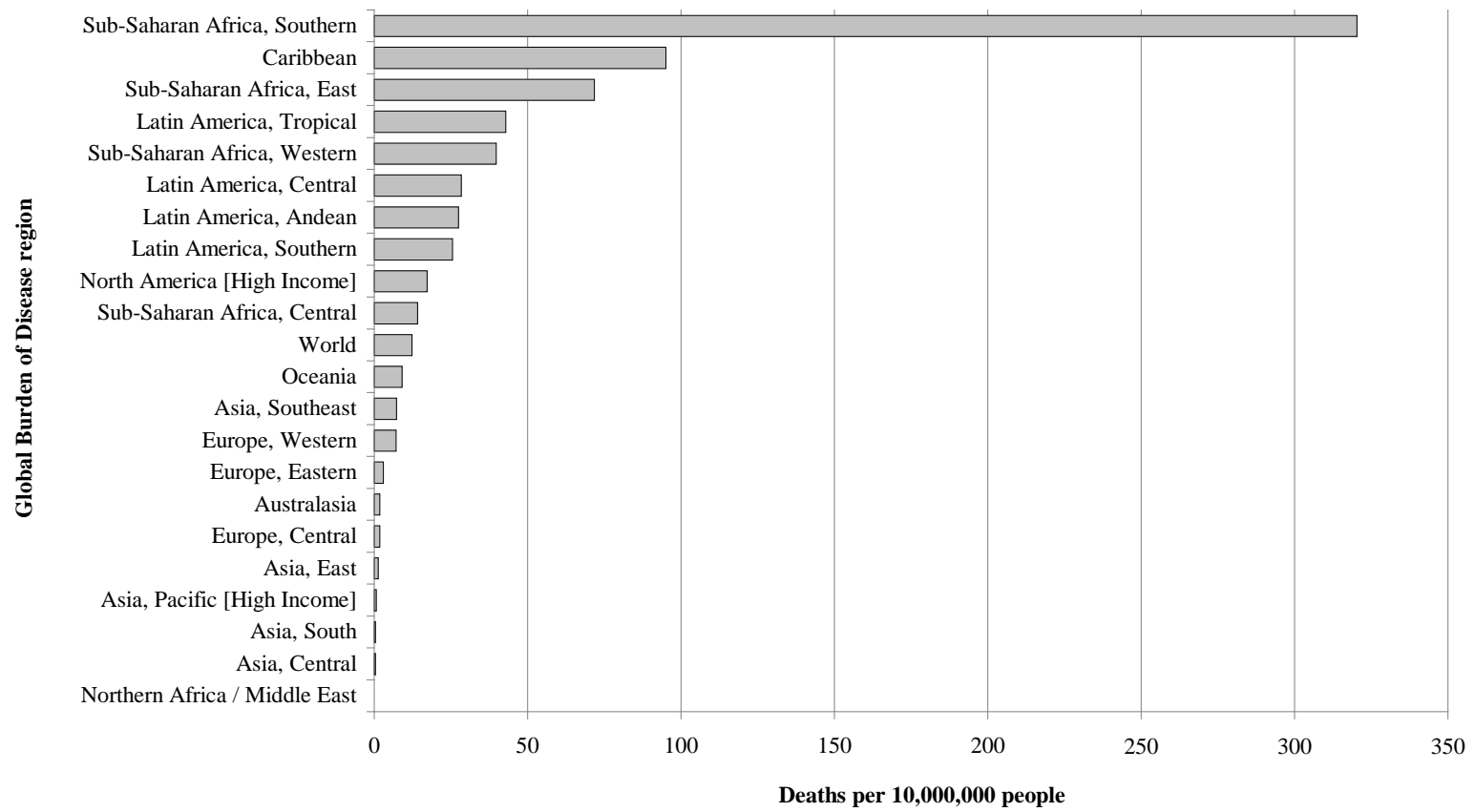

Figure 2

Deaths attributable to HIV/AIDS from not adhering to antiretroviral therapy because of heavy alcohol consumption by Global Burden of Disease region for 2004 per 10,000,000 people 15 years or older

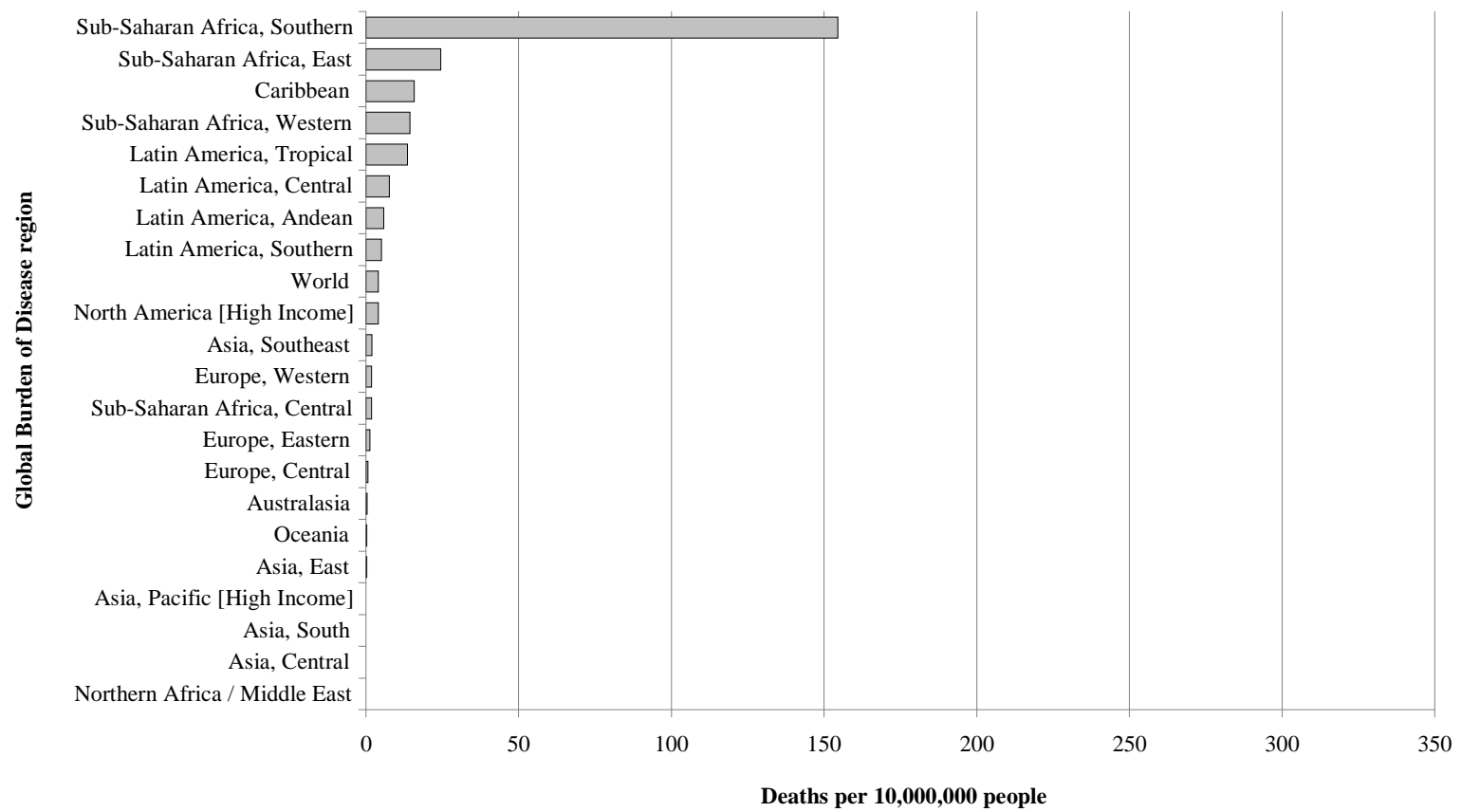


Figure 3

Potential years of life lost attributable to HIV/AIDS from not adhering to antiretroviral therapy because of alcohol consumption by Global Burden of Disease region for 2004 per 10,000,000 people 15 years or older

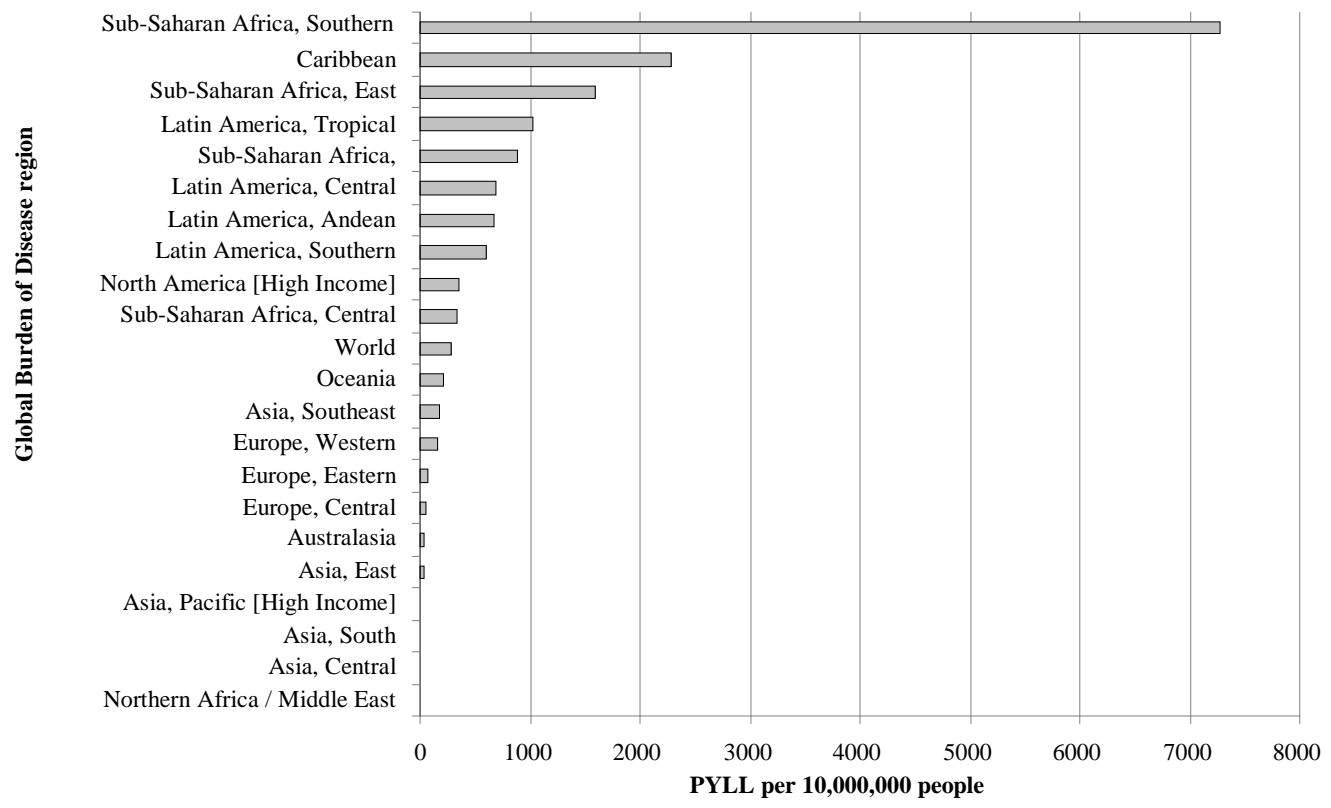

Figure 4

Potential years of life lost attributable to HIV/AIDS from not adhering to antiretroviral therapy because of heavy alcohol consumption by Global Burden of Disease region for 2004 per 10,000,000 people 15 years or older

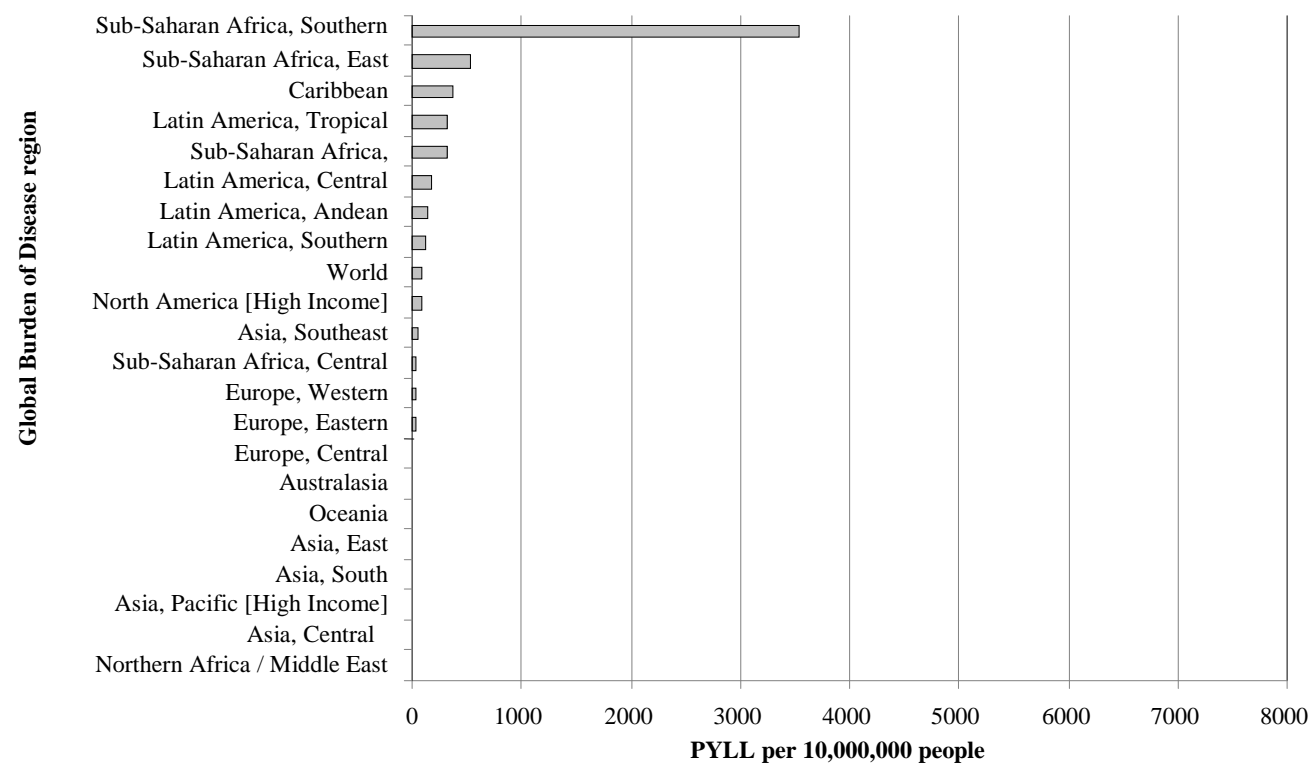




\section{Table 5a}

Potential years of life lost to HIV/AIDS from not adhering to antiretroviral therapy because of alcohol consumption by Global Burden of Disease region for 2004 (Men)

\begin{tabular}{|c|c|c|c|c|c|c|c|c|c|}
\hline \multirow[b]{2}{*}{$\begin{array}{l}\text { Global Burden of } \\
\text { Disease region }\end{array}$} & \multicolumn{3}{|c|}{ 15-34 years } & \multicolumn{3}{|c|}{ 35-64 years } & \multicolumn{3}{|c|}{$65+$ years } \\
\hline & $\begin{array}{c}\text { Point } \\
\text { estimate }\end{array}$ & $\begin{array}{c}\text { Lower } \\
\text { estimate }\end{array}$ & $\begin{array}{c}\text { Upper } \\
\text { estimate }\end{array}$ & $\begin{array}{c}\text { Point } \\
\text { estimate }\end{array}$ & $\begin{array}{c}\text { Lower } \\
\text { estimate }\end{array}$ & $\begin{array}{c}\text { Upper } \\
\text { estimate }\end{array}$ & $\begin{array}{c}\text { Point } \\
\text { estimate }\end{array}$ & $\begin{array}{c}\text { Lower } \\
\text { estimate }\end{array}$ & $\begin{array}{c}\text { Upper } \\
\text { estimate }\end{array}$ \\
\hline $\begin{array}{l}\text { Asia, Pacific } \\
\quad \text { [High Income] }\end{array}$ & 91 & 47 & 135 & 161 & 83 & 239 & 5 & 3 & 8 \\
\hline Asia, Central & 32 & 12 & 52 & 30 & 12 & 48 & 0 & 0 & 0 \\
\hline Asia, East & 1,911 & 814 & 3,008 & 2,357 & 1,012 & 3,702 & 11 & 5 & 18 \\
\hline Asia, South & 732 & 104 & 1,361 & 735 & 177 & 1,293 & 2 & 0 & 3 \\
\hline Asia, Southeast & 5,338 & 1,827 & 8,849 & 4,290 & 1,318 & 7,261 & 19 & 5 & 32 \\
\hline Australasia & 23 & 11 & 36 & 65 & 32 & 99 & 3 & 1 & 5 \\
\hline Caribbean & 2,511 & 1,143 & 3,878 & 3,505 & 1,587 & 5,423 & 27 & 11 & 44 \\
\hline Europe, Central & 222 & 108 & 337 & 190 & 94 & 287 & 4 & 2 & 6 \\
\hline Europe, Eastern & 700 & 270 & 1,130 & 639 & 244 & 1,033 & 3 & 1 & 5 \\
\hline Europe, Western & 1,605 & 804 & 2,406 & 3,840 & 1,939 & 5,740 & 107 & 53 & 160 \\
\hline $\begin{array}{l}\text { Latin America, } \\
\text { Andean }\end{array}$ & 1,281 & 576 & 1,986 & 1,326 & 610 & 2,041 & 3 & 1 & 4 \\
\hline $\begin{array}{l}\text { Latin America, } \\
\text { Central }\end{array}$ & 5,318 & 2,390 & 8,246 & 6,203 & 2,903 & 9,503 & 53 & 23 & 84 \\
\hline $\begin{array}{l}\text { Latin America, } \\
\text { Southern }\end{array}$ & 1,148 & 591 & 1,706 & 1,599 & 815 & 2,384 & 31 & 15 & 47 \\
\hline $\begin{array}{l}\text { Latin America, } \\
\text { Tropical }\end{array}$ & 6,767 & 3,356 & 10,177 & 7,336 & 3,455 & 11,218 & 61 & 24 & 99 \\
\hline $\begin{array}{l}\text { Northern Africa / } \\
\text { Middle East }\end{array}$ & 42 & 0 & 83 & 56 & 9 & 103 & 0 & 0 & 0 \\
\hline $\begin{array}{l}\text { North America } \\
\text { [High Income] }\end{array}$ & 2,462 & 1,209 & 3,716 & 7,071 & 3,441 & 10,702 & 158 & 73 & 244 \\
\hline Oceania & 46 & 20 & 71 & 67 & 29 & 105 & 0 & 0 & 1 \\
\hline $\begin{array}{l}\text { Sub-Saharan } \\
\text { Africa, Central }\end{array}$ & 399 & 129 & 669 & 585 & 189 & 980 & 3 & 1 & 5 \\
\hline $\begin{array}{l}\text { Sub-Saharan } \\
\text { Africa, East }\end{array}$ & 4,801 & 1,414 & 8,187 & 12,258 & 4,340 & 20,177 & 180 & 63 & 298 \\
\hline $\begin{array}{l}\text { Sub-Saharan } \\
\text { Africa, Southern }\end{array}$ & 10,299 & 3,755 & 16,844 & 17,769 & 6,478 & 29,059 & 153 & 51 & 255 \\
\hline $\begin{array}{l}\text { Sub-Saharan } \\
\text { Africa, Western }\end{array}$ & 3,118 & 1,074 & 5,163 & 6,934 & 2,630 & 11,239 & 84 & 30 & 139 \\
\hline World & 48,847 & 19,654 & 78,039 & 77,016 & 31,396 & 122,637 & 908 & 361 & 1,455 \\
\hline
\end{tabular}


Table 5b

Potential years of life lost to HIV/AIDS from not adhering to antiretroviral therapy because of alcohol consumption by Global Burden of Disease region for 2004 (Women)

\begin{tabular}{|c|c|c|c|c|c|c|c|c|c|}
\hline \multirow[b]{2}{*}{$\begin{array}{l}\text { Global Burden of } \\
\text { Disease region }\end{array}$} & \multicolumn{3}{|c|}{ 15-34 years } & \multicolumn{3}{|c|}{ 35-64 years } & \multicolumn{3}{|c|}{$65+$ years } \\
\hline & $\begin{array}{c}\begin{array}{c}\text { Point } \\
\text { estimate }\end{array} \\
\end{array}$ & $\begin{array}{c}\text { Lower } \\
\text { estimate }\end{array}$ & $\begin{array}{c}\text { Upper } \\
\text { estimate }\end{array}$ & $\begin{array}{c}\text { Point } \\
\text { estimate }\end{array}$ & $\begin{array}{c}\text { Lower } \\
\text { estimate }\end{array}$ & $\begin{array}{c}\text { Upper } \\
\text { estimate }\end{array}$ & $\begin{array}{c}\begin{array}{c}\text { Point } \\
\text { estimate }\end{array} \\
\end{array}$ & $\begin{array}{c}\begin{array}{c}\text { Lower } \\
\text { estimate }\end{array} \\
\end{array}$ & $\begin{array}{c}\text { Upper } \\
\text { estimate }\end{array}$ \\
\hline $\begin{array}{l}\text { Asia, Pacific } \\
\text { [High Income] }\end{array}$ & 26 & 13 & 39 & 20 & 10 & 30 & 0 & 0 & 1 \\
\hline Asia, Central & 5 & 2 & 8 & 5 & 2 & 8 & 0 & 0 & 0 \\
\hline Asia, East & 340 & 126 & 554 & 316 & 117 & 515 & 2 & 1 & 4 \\
\hline Asia, South & 52 & 0 & 134 & 32 & 0 & 76 & 0 & 0 & 0 \\
\hline Asia, Southeast & 443 & 3 & 883 & 222 & 0 & 486 & 1 & 0 & 3 \\
\hline Australasia & 5 & 3 & 8 & 6 & 3 & 10 & 0 & 0 & 0 \\
\hline Caribbean & 1,282 & 540 & 2,025 & 795 & 282 & 1,309 & 4 & 0 & 7 \\
\hline Europe, Central & 138 & 67 & 209 & 54 & 26 & 83 & 0 & 0 & 0 \\
\hline Europe, Eastern & 161 & 59 & 264 & 121 & 44 & 197 & 1 & 0 & 2 \\
\hline Europe, Western & 543 & 271 & 816 & 949 & 475 & 1,424 & 18 & 8 & 28 \\
\hline $\begin{array}{l}\text { Latin America, } \\
\text { Andean }\end{array}$ & 304 & 128 & 480 & 229 & 97 & 362 & 2 & 1 & 3 \\
\hline $\begin{array}{l}\text { Latin America, } \\
\text { Central }\end{array}$ & 1,217 & 497 & 1,938 & 892 & 357 & 1,426 & 9 & 3 & 15 \\
\hline $\begin{array}{l}\text { Latin America, } \\
\text { Southern }\end{array}$ & 369 & 185 & 553 & 326 & 158 & 495 & 6 & 3 & 9 \\
\hline $\begin{array}{l}\text { Latin America, } \\
\text { Tropical }\end{array}$ & 2,992 & 1,397 & 4,588 & 2,742 & 1,179 & 4,305 & 27 & 9 & 44 \\
\hline $\begin{array}{l}\text { Northern Africa / } \\
\text { Middle East }\end{array}$ & 6 & 0 & 14 & 1 & 0 & 4 & 0 & 0 & 0 \\
\hline $\begin{array}{l}\text { North America } \\
\text { [High Income] }\end{array}$ & 1,141 & 552 & 1,731 & 2,386 & 1,135 & 3,637 & 31 & 13 & 50 \\
\hline Oceania & 14 & 5 & 23 & 18 & 7 & 28 & 0 & 0 & 0 \\
\hline $\begin{array}{l}\text { Sub-Saharan } \\
\text { Africa, Central }\end{array}$ & 511 & 142 & 881 & 469 & 122 & 815 & 2 & 0 & 4 \\
\hline $\begin{array}{l}\text { Sub-Saharan } \\
\text { Africa, East }\end{array}$ & 6,221 & 1,356 & 11,085 & 10,818 & 3,204 & 18,433 & 82 & 22 & 142 \\
\hline $\begin{array}{l}\text { Sub-Saharan } \\
\text { Africa, Southern }\end{array}$ & 6,118 & 1,065 & 11,171 & 8,673 & 1,944 & 15,402 & 55 & 6 & 105 \\
\hline $\begin{array}{l}\text { Sub-Saharan } \\
\text { Africa, Western }\end{array}$ & 3,351 & 890 & 5,812 & 5,414 & 1,767 & 9,061 & 45 & 14 & 76 \\
\hline World & 25,241 & 7,298 & 43,216 & 34,489 & 10,927 & 58,105 & 286 & 80 & 493 \\
\hline
\end{tabular}




\section{References}

Azar, M. M., Springer, S. A., Meyer, J. P., \& Altice, F. L. (2010). A systematic review of the impact of alcohol use disorders on HIV treatment outcomes, adherence to antiretroviral therapy and health care utilization. Drug and Alcohol Dependence, 112, 178-193.

Baliunas, D., Rehm, J., Irving, H., \& Shuper, P. (2010). Alcohol consumption and risk of incident human immunodeficiency virus infection: A meta-analysis. International Journal of Public Health, 55, 159-166.

Braithwaite, R. S., Conigliaro, J., McGinnis, K. A., Maisto, S. A., Bryant, K., \& Justice, A. C. (2008). Adjusting alcohol quantity for mean consumption and intoxication threshold improves prediction of nonadherence in HIV patients and HIV-negative controls. Alcoholism: Clinical and Experimental Research, 32, 1645-1651.

Braithwaite, R. S., Conigliaro, J., Roberts, M. S., Shechter, S., Schaefer, A., McGinnis K., ... Justice, A. C. (2007). Estimating the impact of alcohol consumption on survival for HIV+ individuals. AIDS Care, 19, 459-466.

Braithwaite, R. S., McGinnis, K. A., Conigliaro, J., Maisto, S., Crystal, S., Day, N., ... Justice, A. C. (2005). A temporal and dose-response association between alcohol consumption and medication adherence among veterans in care. Alcoholism: Clinical and Experimental Research, 29, 1190-1197.

Brigido, L. F. M., Rodrigues, R., Casseb, J., Oliveira, D., Rossetti, M., Menezes, P., \& Duarte, A. J. (2001). Impact of adherence to antiretroviral therapy in HIV1-infected patients at a university public service in Brazil. AIDS Patient Care and STDs, 15, 587-593.

Dingle, G. A., \& Oei, T. P. (1997). Is alcohol a cofactor of HIV and AIDS? Evidence from immunological and behavioral studies. Psychological Bulletin, 122, 5671.

Fisher, J. C., Bang, H., \& Kapiga, S. H. (2007). The association between HIV infection and alcohol use: A systematic review and meta-analysis of African studies. Sexually Transmitted Diseases, 34, 856-863.

Garcia de Olalla, P., Knobel, H., Carmona, A., Guelar, A., Lopez-Colomes, J. L., \& Cayla, J. A. (2002). Impact of adherence and highly active antiretroviral therapy on survival in HIV-infected patients. Journal of Acquired Immune Deficiency Syndromes, 30, 105110.

Gmel, G., Shield, K., \& Rehm, J. (2010). Developing a methodology to derive alcohol-attributable fractions for HIV/AIDS mortality based on alcohol's impact on adherence to antiretroviral medication. Population Health Metrics, 9.

Hahn, J. A., \& Samet, J. H. (2010). Alcohol and HIV disease progression: Weighing the evidence. Current HIV/AIDS Reports, 7, 226-233.

Hendershot, C. S., Stoner, S. A., Pantalone, D. W., \& Simoni, J. M. (2009). Alcohol use and antiretroviral adherence: Review and meta-analysis. Journal of Acquired Immune Deficiency Syndromes, 52, 180202.
Institute for Health Metrics and Evaluation. (2010). Global Burden of Diseases: Injuries and risk factors study operations manual. Seattle, WA, United States: Author.

Kehoe, T., Gmel, G., Shield, K. D., Gmel, G., \& Rehm, J. (2012). Determining the best population-level alcohol consumption model and its impact on estimates of alcohol-attributable harms. Population Health Metrics, 10.

Kalichman, S. C., Amaral, C. M., White, D., Swetsze, C., Pope, H., Kalichman, M. O., ... Eaton, L. (2009). Prevalence and clinical implications of interactive toxicity beliefs regarding mixing alcohol and antiretroviral therapies among people living with HIV/AIDS. AIDS Patient Care STDS, 23, 449-454.

Lima, V. D., Harrigan, R., Bangsberg, D. R., Hogg, R. S., Gross, R., Yip, B., ... Julio, S. G. (2009). The combined effect of modern highly active antiretroviral therapy regimens and adherence on mortality over time. Journal of Acquired Immune Deficiency Syndromes, 50, 529-536.

Lima, V. D., Harrigan, R., Murray, M., Moore, D. M., Wood, E., Hogg, R. S., ... Julio, S. C. (2008). Differential impact of adherence on long-term treatment response among naïve HIV-infected individuals. AIDS, 22, 2371-2380.

Lopez, A. D., Mathers, C. D., Ezzati, M., Jamison, D. T., \& Murray, C. J. L. (2006a). Global burden of disease and risk factors. New York, NY \& Washington, DC, United States: The World Bank and Oxford University Press.

Lopez, A. D., Mathers, C. D., Ezzati, M., Jamison, D. T., \& Murray, C. J. L. (2006b). Global and regional burden of disease and risk factors, 2001: Systematic analysis of population health data. Lancet, 367, 1747-1757.

Marcellin, F., Boyer, S., Potopopescu, C., Dia, A., OngoloZogo, P., Koulla-Shiro, S., ... Carrier, M. P. (2008). Determinants of unplanned antiretroviral treatment interruptions among people living with HIV in Yaoundé, Cameroon. Tropical Medicine \& International Health, 13, 1470-1478.

McKirnan, D. J., Ostrow, D. G., \& Hope, B. (1996). Sex, drugs and escape: A psychological model of HIV-risk sexual behaviours. AIDS Care, 8, 655-669.

Nachega, J. B., Hislop, M., Dowdy, D. W., Lo, M., Omer, S. B., Regensberg, L., ... Maartens, G. (2006). Adherence to highly active antiretroviral therapy assessed by pharmacy claims predicts survival in HIVinfected South African adults. Journal of Acquired Immune Deficiency Syndromes, 43, 78-84.

Nemeroff, C. J., Hoyt, M. A., Huebner, D. M., \& Proescholdbell, R. J. (2008). The cognitive escape scale: Measuring HIV-related thought avoidance. AIDS and Behavior, 12, 305-320.

Neuman, M. G., Monteiro, M., \& Rehm J. (2006). Drug interactions between psychoactive substances and antiretroviral therapy in individuals infected with human immunodeficiency and hepatitis viruses. Substance Use and Misuse, 41, 1395-1463.

Parsons, J. T., Rosof, E., \& Mustanski, B. (2008). The temporal relationship between alcohol consumption and HIV-medication adherence: A multilevel model of 
direct and moderating effects. Health Psychology, 27, 628-637.

Rehm, J., Baliunas, D., Borges, G. L. G., Graham, K., Irving, H. M., Kehoe, T., .. . Taylor, B. (2010). The relation between different dimensions of alcohol consumption and burden of disease-An overview. Addiction, 105, 817-843.

Rehm, J., Shield, K. D., Joharchi, N., \& Shuper, P. A. (2012). Alcohol consumption and the intention to engage in unprotected sex: Systematic review and meta-analysis of experimental studies. Addiction, 107, 51-59.

Sankar, A., Wunderlich, T., Neufeld, S., \& Luborsky, M. (2007). Sero-positive African Americans' beliefs about alcohol and their impact on anti-retroviral adherence. AIDS and Behavior, 11, 195-203.

Shield, K., \& Rehm, J. (2012). Difficulties with telephonebased surveys on alcohol in high-income countries: The Canadian example. International Journal of Methods in Psychiatric Research, 21, 17-28.

Shuper, P. A., Joharchi, N., Irving, H., \& Rehm, J. (2009). Alcohol as a correlate of unprotected sexual behavior among people living with HIV/AIDS: Review and meta-analysis. AIDS and Behaviour, 13, 1021-1036.
Shuper, P. A., Neuman, M., Kanteres, F., Baliunas, D., Joharchi, N., \& Rehm, J. (2010). Causal considerations on alcohol and HIV/AIDS-A systematic review. Alcohol and Alcoholism, 45, 159166.

Stall, R., \& Leigh, B. (1994). Understanding the relationship between drug or alcohol use and high risk sexual activity for HIV transmission: Where do we go from here? Addiction, 89, 131-134.

UNAIDS. (2006). Report on the global AIDS epidemic. Geneva, Switzerland: UNAIDS-Joint United Nations Programme on HIV/AIDS. Geneva, Switzerland. Retrieved from http://www.unaids.org/en/Knowledge Centre/HIVData/GlobalReport/2006/default.asp.

United Nations Population Division. (2010). World populations prospects-The 2008 revision. New York, NY, United States: United Nations.

World Health Organization. (2008). The global burden of disease: 2004 update. Geneva, Switzerland: Author.

World Health Organization. (2009). Global health risks. Mortality and burden of disease attributable to selected major risks. Geneva, Switzerland: Author.

World Health Organization. (2011). Global status report on alcohol and health. Geneva, Switzerland: Author.

\section{Appendices}

\section{Appendix Table 1}

Prevalence of people who are receiving antiretroviral therapy out of those who are also in need of antiretroviral therapy by region

\begin{tabular}{lccc}
\hline & Point estimate & Lower estimate & Upper estimate \\
\hline Asia, Pacific [High Income] & $80.00 \%$ & $77.52 \%$ & $82.48 \%$ \\
Asia, Central & $14.29 \%$ & $12.12 \%$ & $16.46 \%$ \\
Asia, East & $24.56 \%$ & $21.89 \%$ & $27.23 \%$ \\
Asia, South & $5.63 \%$ & $4.20 \%$ & $7.06 \%$ \\
Asia, Southeast & $24.55 \%$ & $21.88 \%$ & $27.22 \%$ \\
Australasia & $60.38 \%$ & $57.35 \%$ & $63.42 \%$ \\
Caribbean & $49.19 \%$ & $46.09 \%$ & $52.29 \%$ \\
Europe, Central & $68.45 \%$ & $65.57 \%$ & $71.33 \%$ \\
Europe, Eastern & $7.49 \%$ & $5.86 \%$ & $9.12 \%$ \\
Europe, Western & $71.85 \%$ & $69.07 \%$ & $74.64 \%$ \\
Latin America, Andean & $46.58 \%$ & $43.49 \%$ & $49.67 \%$ \\
Latin America, Central & $62.98 \%$ & $59.99 \%$ & $65.97 \%$ \\
Latin America, Southern & $78.64 \%$ & $76.10 \%$ & $81.18 \%$ \\
Latin America, Tropical & $81.35 \%$ & $78.93 \%$ & $83.76 \%$ \\
Northern Africa / Middle East & $17.36 \%$ & $15.01 \%$ & $19.71 \%$ \\
North America [High Income] & $70.10 \%$ & $67.26 \%$ & $72.94 \%$ \\
Oceania & $26.75 \%$ & $24.01 \%$ & $29.49 \%$ \\
Sub-Saharan Africa, Central & $4.25 \%$ & $3.00 \%$ & $5.50 \%$ \\
Sub-Saharan Africa, East & $14.24 \%$ & $12.07 \%$ & $16.41 \%$ \\
Sub-Saharan Africa, Southern & $21.67 \%$ & $19.11 \%$ & $24.22 \%$ \\
Sub-Saharan Africa, Western & $13.47 \%$ & $11.36 \%$ & $15.59 \%$ \\
World & $30.87 \%$ & $28.56 \%$ & $33.18 \%$ \\
\hline
\end{tabular}




\section{Appendix Table 2a}

Alcohol-attributable fractions for HIV from not adhering to antiretroviral therapy because of alcohol consumption, and heavy alcohol consumption, by Global Burden of Disease region for 2004 (Men)

\begin{tabular}{|c|c|c|c|c|c|c|c|c|c|}
\hline \multirow[b]{2}{*}{$\begin{array}{l}\text { Global Burden of } \\
\text { Disease region }\end{array}$} & \multicolumn{3}{|c|}{ 15-34 years } & \multicolumn{3}{|c|}{ 35-64 years } & \multicolumn{3}{|c|}{$65+$ years } \\
\hline & $\begin{array}{c}\begin{array}{c}\text { Point } \\
\text { estimate }\end{array} \\
\end{array}$ & $\begin{array}{c}\text { Lower } \\
\text { estimate }\end{array}$ & $\begin{array}{c}\text { Upper } \\
\text { estimate }\end{array}$ & $\begin{array}{c}\begin{array}{c}\text { Point } \\
\text { estimate }\end{array} \\
\end{array}$ & $\begin{array}{c}\begin{array}{c}\text { Lower } \\
\text { estimate }\end{array} \\
\end{array}$ & $\begin{array}{c}\begin{array}{c}\text { Upper } \\
\text { estimate }\end{array} \\
\end{array}$ & $\begin{array}{c}\begin{array}{c}\text { Point } \\
\text { estimate }\end{array} \\
\end{array}$ & $\begin{array}{c}\text { Lower } \\
\text { estimate }\end{array}$ & $\begin{array}{c}\text { Upper } \\
\text { estimate }\end{array}$ \\
\hline $\begin{array}{l}\text { Asia, Pacific } \\
\text { [High Income] }\end{array}$ & $7.42 \%$ & $3.84 \%$ & $11.01 \%$ & $7.23 \%$ & $3.72 \%$ & $10.74 \%$ & $6.02 \%$ & $2.99 \%$ & $9.06 \%$ \\
\hline Asia, Central & $0.50 \%$ & $0.19 \%$ & $0.80 \%$ & $0.63 \%$ & $0.26 \%$ & $1.01 \%$ & $0.47 \%$ & $0.18 \%$ & $0.76 \%$ \\
\hline Asia, East & $1.10 \%$ & $0.47 \%$ & $1.73 \%$ & $1.14 \%$ & $0.49 \%$ & $1.80 \%$ & $1.02 \%$ & $0.43 \%$ & $1.61 \%$ \\
\hline Asia, South & $0.05 \%$ & $0.01 \%$ & $0.09 \%$ & $0.08 \%$ & $0.02 \%$ & $0.14 \%$ & $0.05 \%$ & $0.01 \%$ & $0.09 \%$ \\
\hline Asia, Southeast & $0.55 \%$ & $0.19 \%$ & $0.90 \%$ & $0.44 \%$ & $0.13 \%$ & $0.74 \%$ & $0.37 \%$ & $0.10 \%$ & $0.63 \%$ \\
\hline Australasia & $4.32 \%$ & $2.10 \%$ & $6.53 \%$ & $4.41 \%$ & $2.15 \%$ & $6.67 \%$ & $3.91 \%$ & $1.87 \%$ & $5.96 \%$ \\
\hline Caribbean & $2.66 \%$ & $1.21 \%$ & $4.11 \%$ & $2.59 \%$ & $1.17 \%$ & $4.01 \%$ & $1.52 \%$ & $0.59 \%$ & $2.45 \%$ \\
\hline Europe, Central & $4.73 \%$ & $2.30 \%$ & $7.16 \%$ & $5.05 \%$ & $2.49 \%$ & $7.62 \%$ & $4.50 \%$ & $2.16 \%$ & $6.84 \%$ \\
\hline Europe, Eastern & $0.31 \%$ & $0.12 \%$ & $0.51 \%$ & $0.30 \%$ & $0.12 \%$ & $0.49 \%$ & $0.26 \%$ & $0.10 \%$ & $0.43 \%$ \\
\hline Europe, Western & $5.74 \%$ & $2.88 \%$ & $8.61 \%$ & $6.01 \%$ & $3.04 \%$ & $8.99 \%$ & $5.37 \%$ & $2.65 \%$ & $8.08 \%$ \\
\hline $\begin{array}{l}\text { Latin America, } \\
\text { Andean }\end{array}$ & $2.40 \%$ & $1.08 \%$ & $3.72 \%$ & $2.67 \%$ & $1.23 \%$ & $4.12 \%$ & $0.92 \%$ & $0.29 \%$ & $1.55 \%$ \\
\hline $\begin{array}{l}\text { Latin America, } \\
\text { Central }\end{array}$ & $3.13 \%$ & $1.41 \%$ & $4.86 \%$ & $3.71 \%$ & $1.74 \%$ & $5.69 \%$ & $2.74 \%$ & $1.19 \%$ & $4.30 \%$ \\
\hline $\begin{array}{l}\text { Latin America, } \\
\text { Southern }\end{array}$ & $7.11 \%$ & $3.66 \%$ & $10.57 \%$ & $6.74 \%$ & $3.43 \%$ & $10.05 \%$ & $5.76 \%$ & $2.84 \%$ & $8.68 \%$ \\
\hline $\begin{array}{l}\text { Latin America, } \\
\text { Tropical }\end{array}$ & $6.11 \%$ & $3.03 \%$ & $9.19 \%$ & $4.90 \%$ & $2.31 \%$ & $7.49 \%$ & $2.76 \%$ & $1.07 \%$ & $4.45 \%$ \\
\hline $\begin{array}{l}\text { Northern Africa / } \\
\text { Middle East }\end{array}$ & $0.09 \%$ & $0.00 \%$ & $0.19 \%$ & $0.15 \%$ & $0.02 \%$ & $0.28 \%$ & $0.07 \%$ & $0.00 \%$ & $0.15 \%$ \\
\hline $\begin{array}{l}\text { North America } \\
\text { [High Income] }\end{array}$ & $5.09 \%$ & $2.50 \%$ & $7.69 \%$ & $4.87 \%$ & $2.37 \%$ & $7.37 \%$ & $3.83 \%$ & $1.76 \%$ & $5.89 \%$ \\
\hline Oceania & $1.37 \%$ & $0.60 \%$ & $2.14 \%$ & $1.31 \%$ & $0.57 \%$ & $2.04 \%$ & $1.19 \%$ & $0.51 \%$ & $1.88 \%$ \\
\hline $\begin{array}{l}\text { Sub-Saharan } \\
\text { Africa, Central }\end{array}$ & $0.13 \%$ & $0.04 \%$ & $0.21 \%$ & $0.13 \%$ & $0.04 \%$ & $0.22 \%$ & $0.06 \%$ & $0.01 \%$ & $0.10 \%$ \\
\hline $\begin{array}{l}\text { Sub-Saharan } \\
\text { Africa, East }\end{array}$ & $0.24 \%$ & $0.07 \%$ & $0.41 \%$ & $0.36 \%$ & $0.13 \%$ & $0.60 \%$ & $0.35 \%$ & $0.12 \%$ & $0.57 \%$ \\
\hline $\begin{array}{l}\text { Sub-Saharan } \\
\text { Africa, Southern }\end{array}$ & $0.57 \%$ & $0.21 \%$ & $0.93 \%$ & $0.57 \%$ & $0.21 \%$ & $0.93 \%$ & $0.45 \%$ & $0.15 \%$ & $0.76 \%$ \\
\hline $\begin{array}{l}\text { Sub-Saharan } \\
\text { Africa, Western }\end{array}$ & $0.32 \%$ & $0.11 \%$ & $0.53 \%$ & $0.43 \%$ & $0.16 \%$ & $0.69 \%$ & $0.35 \%$ & $0.13 \%$ & $0.58 \%$ \\
\hline
\end{tabular}




\section{Appendix Table 2b}

Alcohol-attributable fractions for HIV from not adhering to antiretroviral therapy because of alcohol consumption, and heavy alcohol consumption, by Global Burden of Disease region for 2004 (Women)

\begin{tabular}{|c|c|c|c|c|c|c|c|c|c|}
\hline \multirow[b]{2}{*}{$\begin{array}{l}\text { Global Burden of } \\
\text { Disease region }\end{array}$} & \multicolumn{3}{|c|}{$15-34$ years } & \multicolumn{3}{|c|}{$35-64$ years } & \multicolumn{3}{|c|}{$65+$ years } \\
\hline & $\begin{array}{c}\begin{array}{c}\text { Point } \\
\text { estimate }\end{array} \\
\end{array}$ & $\begin{array}{c}\text { Lower } \\
\text { estimate }\end{array}$ & $\begin{array}{c}\text { Upper } \\
\text { estimate }\end{array}$ & $\begin{array}{c}\begin{array}{c}\text { Point } \\
\text { estimate }\end{array} \\
\end{array}$ & $\begin{array}{c}\text { Lower } \\
\text { estimate }\end{array}$ & $\begin{array}{c}\begin{array}{c}\text { Upper } \\
\text { estimate }\end{array} \\
\end{array}$ & $\begin{array}{c}\begin{array}{c}\text { Point } \\
\text { estimate }\end{array} \\
\end{array}$ & $\begin{array}{c}\text { Lower } \\
\text { estimate }\end{array}$ & $\begin{array}{c}\begin{array}{c}\text { Upper } \\
\text { estimate }\end{array} \\
\end{array}$ \\
\hline $\begin{array}{l}\text { Asia, Pacific } \\
\text { [High Income] }\end{array}$ & $7.15 \%$ & $3.67 \%$ & $10.62 \%$ & $6.61 \%$ & $3.35 \%$ & $9.88 \%$ & $4.58 \%$ & $2.13 \%$ & $7.02 \%$ \\
\hline Asia, Central & $0.33 \%$ & $0.11 \%$ & $0.54 \%$ & $0.56 \%$ & $0.23 \%$ & $0.90 \%$ & $0.32 \%$ & $0.11 \%$ & $0.53 \%$ \\
\hline Asia, East & $0.66 \%$ & $0.25 \%$ & $1.08 \%$ & $0.66 \%$ & $0.25 \%$ & $1.08 \%$ & $0.54 \%$ & $0.19 \%$ & $0.90 \%$ \\
\hline Asia, South & $0.01 \%$ & $0.00 \%$ & $0.03 \%$ & $0.01 \%$ & $0.00 \%$ & $0.03 \%$ & $0.00 \%$ & $0.00 \%$ & $0.01 \%$ \\
\hline Asia, Southeast & $0.14 \%$ & $0.00 \%$ & $0.28 \%$ & $0.09 \%$ & $0.00 \%$ & $0.19 \%$ & $0.04 \%$ & $0.00 \%$ & $0.10 \%$ \\
\hline Australasia & $4.15 \%$ & $2.00 \%$ & $6.30 \%$ & $4.28 \%$ & $2.08 \%$ & $6.48 \%$ & $3.09 \%$ & $1.39 \%$ & $4.78 \%$ \\
\hline Caribbean & $1.94 \%$ & $0.82 \%$ & $3.06 \%$ & $1.21 \%$ & $0.43 \%$ & $1.99 \%$ & $0.41 \%$ & $0.04 \%$ & $0.78 \%$ \\
\hline Europe, Central & $4.65 \%$ & $2.25 \%$ & $7.05 \%$ & $4.29 \%$ & $2.04 \%$ & $6.54 \%$ & $1.35 \%$ & $0.40 \%$ & $2.30 \%$ \\
\hline Europe, Eastern & $0.25 \%$ & $0.09 \%$ & $0.41 \%$ & $0.24 \%$ & $0.09 \%$ & $0.40 \%$ & $0.15 \%$ & $0.05 \%$ & $0.26 \%$ \\
\hline Europe, Western & $5.62 \%$ & $2.80 \%$ & $8.44 \%$ & $5.70 \%$ & $2.85 \%$ & $8.55 \%$ & $3.93 \%$ & $1.81 \%$ & $6.05 \%$ \\
\hline $\begin{array}{l}\text { Latin America, } \\
\text { Andean }\end{array}$ & $1.83 \%$ & $0.77 \%$ & $2.89 \%$ & $1.88 \%$ & $0.79 \%$ & $2.96 \%$ & $1.12 \%$ & $0.39 \%$ & $1.85 \%$ \\
\hline $\begin{array}{l}\text { Latin America, } \\
\text { Central }\end{array}$ & $2.27 \%$ & $0.93 \%$ & $3.62 \%$ & $2.16 \%$ & $0.86 \%$ & $3.45 \%$ & $1.71 \%$ & $0.62 \%$ & $2.80 \%$ \\
\hline $\begin{array}{l}\text { Latin America, } \\
\text { Southern }\end{array}$ & $6.27 \%$ & $3.15 \%$ & $9.39 \%$ & $5.24 \%$ & $2.53 \%$ & $7.95 \%$ & $4.53 \%$ & $2.11 \%$ & $6.95 \%$ \\
\hline $\begin{array}{l}\text { Latin America, } \\
\text { Tropical }\end{array}$ & $4.73 \%$ & $2.21 \%$ & $7.26 \%$ & $3.60 \%$ & $1.55 \%$ & $5.66 \%$ & $2.01 \%$ & $0.66 \%$ & $3.37 \%$ \\
\hline $\begin{array}{l}\text { Northern Africa / } \\
\text { Middle East }\end{array}$ & $0.05 \%$ & $0.00 \%$ & $0.11 \%$ & $0.01 \%$ & $0.00 \%$ & $0.05 \%$ & $0.01 \%$ & $0.00 \%$ & $0.02 \%$ \\
\hline $\begin{array}{l}\text { North America } \\
\text { [High Income] }\end{array}$ & $4.73 \%$ & $2.29 \%$ & 7.17\% & $4.38 \%$ & $2.08 \%$ & $6.68 \%$ & $2.44 \%$ & $0.98 \%$ & $3.90 \%$ \\
\hline Oceania & $0.70 \%$ & $0.26 \%$ & $1.15 \%$ & $0.89 \%$ & $0.35 \%$ & $1.43 \%$ & $0.82 \%$ & $0.32 \%$ & $1.32 \%$ \\
\hline $\begin{array}{l}\text { Sub-Saharan } \\
\text { Africa, Central }\end{array}$ & $0.09 \%$ & $0.02 \%$ & $0.15 \%$ & $0.08 \%$ & $0.02 \%$ & $0.13 \%$ & $0.03 \%$ & $0.00 \%$ & $0.06 \%$ \\
\hline $\begin{array}{l}\text { Sub-Saharan } \\
\text { Africa, East }\end{array}$ & $0.16 \%$ & $0.04 \%$ & $0.29 \%$ & $0.24 \%$ & $0.07 \%$ & $0.41 \%$ & $0.21 \%$ & $0.06 \%$ & $0.37 \%$ \\
\hline $\begin{array}{l}\text { Sub-Saharan } \\
\text { Africa, Southern }\end{array}$ & $0.21 \%$ & $0.04 \%$ & $0.38 \%$ & $0.25 \%$ & $0.06 \%$ & $0.45 \%$ & $0.16 \%$ & $0.02 \%$ & $0.31 \%$ \\
\hline $\begin{array}{l}\text { Sub-Saharan } \\
\text { Africa, Western }\end{array}$ & $0.19 \%$ & $0.05 \%$ & $0.34 \%$ & $0.28 \%$ & $0.09 \%$ & $0.47 \%$ & $0.25 \%$ & $0.08 \%$ & $0.42 \%$ \\
\hline
\end{tabular}




\section{Appendix Table 3a}

Alcohol-attributable fractions for HIV from not adhering to antiretroviral therapy because of heavy alcohol consumption by Global Burden of Disease region for 2004 (Men)

\begin{tabular}{|c|c|c|c|c|c|c|c|c|c|}
\hline \multirow[b]{2}{*}{$\begin{array}{l}\text { Global Burden of } \\
\text { Disease region }\end{array}$} & \multicolumn{3}{|c|}{ 15-34 years } & \multicolumn{3}{|c|}{ 35-64 years } & \multicolumn{3}{|c|}{$65+$ years } \\
\hline & $\begin{array}{c}\text { Point } \\
\text { estimate }\end{array}$ & $\begin{array}{c}\text { Lower } \\
\text { estimate }\end{array}$ & $\begin{array}{c}\text { Upper } \\
\text { estimate }\end{array}$ & $\begin{array}{c}\text { Point } \\
\text { estimate }\end{array}$ & $\begin{array}{c}\text { Lower } \\
\text { estimate }\end{array}$ & $\begin{array}{c}\text { Upper } \\
\text { estimate }\end{array}$ & $\begin{array}{c}\text { Point } \\
\text { estimate }\end{array}$ & $\begin{array}{c}\text { Lower } \\
\text { estimate }\end{array}$ & $\begin{array}{c}\text { Upper } \\
\text { estimate }\end{array}$ \\
\hline $\begin{array}{l}\text { Asia, Pacific } \\
\text { [High Income] }\end{array}$ & $1.07 \%$ & $0.38 \%$ & $1.75 \%$ & $2.14 \%$ & $0.85 \%$ & $3.43 \%$ & $0.87 \%$ & $0.28 \%$ & $1.45 \%$ \\
\hline Asia, Central & $0.12 \%$ & $0.04 \%$ & $0.20 \%$ & $0.13 \%$ & $0.05 \%$ & $0.20 \%$ & $0.06 \%$ & $0.02 \%$ & $0.10 \%$ \\
\hline Asia, East & $0.03 \%$ & $0.01 \%$ & $0.05 \%$ & $0.30 \%$ & $0.10 \%$ & $0.49 \%$ & $0.17 \%$ & $0.06 \%$ & $0.29 \%$ \\
\hline Asia, South & $0.01 \%$ & $0.01 \%$ & $0.02 \%$ & $0.03 \%$ & $0.01 \%$ & $0.05 \%$ & $0.02 \%$ & $0.01 \%$ & $0.03 \%$ \\
\hline Asia, Southeast & $0.07 \%$ & $0.03 \%$ & $0.10 \%$ & $0.18 \%$ & $0.08 \%$ & $0.29 \%$ & $0.09 \%$ & $0.05 \%$ & $0.14 \%$ \\
\hline Australasia & $0.77 \%$ & $0.27 \%$ & $1.27 \%$ & $1.04 \%$ & $0.39 \%$ & $1.69 \%$ & $0.70 \%$ & $0.26 \%$ & $1.14 \%$ \\
\hline Caribbean & $0.37 \%$ & $0.16 \%$ & $0.58 \%$ & $0.51 \%$ & $0.21 \%$ & $0.81 \%$ & $0.26 \%$ & $0.07 \%$ & $0.44 \%$ \\
\hline Europe, Central & $1.52 \%$ & $0.56 \%$ & $2.48 \%$ & $2.15 \%$ & $0.85 \%$ & $3.44 \%$ & $1.62 \%$ & $0.64 \%$ & $2.59 \%$ \\
\hline Europe, Eastern & $0.15 \%$ & $0.04 \%$ & $0.25 \%$ & $0.14 \%$ & $0.04 \%$ & $0.23 \%$ & $0.09 \%$ & $0.03 \%$ & $0.16 \%$ \\
\hline Europe, Western & $1.41 \%$ & $0.51 \%$ & $2.31 \%$ & $1.83 \%$ & $0.72 \%$ & $2.94 \%$ & $1.13 \%$ & $0.42 \%$ & $1.84 \%$ \\
\hline $\begin{array}{l}\text { Latin America, } \\
\text { Andean }\end{array}$ & $0.43 \%$ & $0.13 \%$ & $0.72 \%$ & $0.70 \%$ & $0.25 \%$ & $1.15 \%$ & $0.08 \%$ & $0.00 \%$ & $0.18 \%$ \\
\hline $\begin{array}{l}\text { Latin America, } \\
\text { Central }\end{array}$ & $0.84 \%$ & $0.33 \%$ & $1.35 \%$ & $1.09 \%$ & $0.47 \%$ & $1.70 \%$ & $0.74 \%$ & $0.29 \%$ & $1.18 \%$ \\
\hline $\begin{array}{l}\text { Latin America, } \\
\text { Southern }\end{array}$ & $1.38 \%$ & $0.51 \%$ & $2.25 \%$ & $1.45 \%$ & $0.53 \%$ & $2.38 \%$ & $0.99 \%$ & $0.33 \%$ & $1.66 \%$ \\
\hline $\begin{array}{l}\text { Latin America, } \\
\text { Tropical }\end{array}$ & $1.98 \%$ & $0.89 \%$ & $3.08 \%$ & $1.78 \%$ & $0.68 \%$ & $2.89 \%$ & $0.44 \%$ & $0.11 \%$ & $0.77 \%$ \\
\hline $\begin{array}{l}\text { Northern Africa / } \\
\text { Middle East }\end{array}$ & $0.04 \%$ & $0.02 \%$ & $0.06 \%$ & $0.03 \%$ & $0.02 \%$ & $0.05 \%$ & $0.00 \%$ & $0.00 \%$ & $0.00 \%$ \\
\hline $\begin{array}{l}\text { North America } \\
\text { [High Income] }\end{array}$ & $1.65 \%$ & $0.68 \%$ & $2.62 \%$ & $1.18 \%$ & $0.48 \%$ & $1.88 \%$ & $0.59 \%$ & $0.22 \%$ & $0.97 \%$ \\
\hline Oceania & $0.03 \%$ & $0.00 \%$ & $0.05 \%$ & $0.05 \%$ & $0.01 \%$ & $0.09 \%$ & $0.01 \%$ & $0.00 \%$ & $0.02 \%$ \\
\hline $\begin{array}{l}\text { Sub-Saharan } \\
\text { Africa, Central }\end{array}$ & $0.01 \%$ & $0.00 \%$ & $0.02 \%$ & $0.02 \%$ & $0.01 \%$ & $0.03 \%$ & $0.00 \%$ & $0.00 \%$ & $0.01 \%$ \\
\hline $\begin{array}{l}\text { Sub-Saharan } \\
\text { Africa, East }\end{array}$ & $0.07 \%$ & $0.03 \%$ & $0.11 \%$ & $0.15 \%$ & $0.06 \%$ & $0.24 \%$ & $0.08 \%$ & $0.04 \%$ & $0.13 \%$ \\
\hline $\begin{array}{l}\text { Sub-Saharan } \\
\text { Africa, Southern }\end{array}$ & $0.28 \%$ & $0.10 \%$ & $0.46 \%$ & $0.28 \%$ & $0.10 \%$ & $0.45 \%$ & $0.12 \%$ & $0.05 \%$ & $0.19 \%$ \\
\hline $\begin{array}{l}\text { Sub-Saharan } \\
\text { Africa, Western }\end{array}$ & $0.10 \%$ & $0.04 \%$ & $0.16 \%$ & $0.17 \%$ & $0.07 \%$ & $0.27 \%$ & $0.06 \%$ & $0.03 \%$ & $0.09 \%$ \\
\hline
\end{tabular}




\section{Appendix Table 3b}

Alcohol-attributable fractions for HIV from not adhering to antiretroviral therapy because of heavy alcohol consumption by Global Burden of Disease region for 2004 (Women)

\begin{tabular}{|c|c|c|c|c|c|c|c|c|c|}
\hline \multirow{2}{*}{$\begin{array}{l}\text { Global Burden } \\
\text { of Disease } \\
\text { region }\end{array}$} & \multicolumn{3}{|c|}{ 15-34 years } & \multicolumn{3}{|c|}{ 35-64 years } & \multicolumn{3}{|c|}{$65+$ years } \\
\hline & $\begin{array}{c}\text { Point } \\
\text { estimate }\end{array}$ & $\begin{array}{l}\text { Lower } \\
\text { estimate }\end{array}$ & $\begin{array}{c}\text { Upper } \\
\text { estimate }\end{array}$ & $\begin{array}{c}\text { Point } \\
\text { estimate }\end{array}$ & $\begin{array}{c}\text { Lower } \\
\text { estimate }\end{array}$ & $\begin{array}{c}\text { Upper } \\
\text { estimate }\end{array}$ & $\begin{array}{c}\text { Point } \\
\text { estimate }\end{array}$ & $\begin{array}{l}\text { Lower } \\
\text { estimate }\end{array}$ & $\begin{array}{c}\text { Upper } \\
\text { estimate }\end{array}$ \\
\hline $\begin{array}{l}\text { Asia, Pacific } \\
\text { [High Income] }\end{array}$ & $0.33 \%$ & $0.04 \%$ & $0.62 \%$ & $0.75 \%$ & $0.19 \%$ & $1.31 \%$ & $0.06 \%$ & $0.00 \%$ & $0.14 \%$ \\
\hline Asia, Central & $0.03 \%$ & $0.00 \%$ & $0.06 \%$ & $0.06 \%$ & $0.02 \%$ & $0.11 \%$ & $0.00 \%$ & $0.00 \%$ & $0.01 \%$ \\
\hline Asia, East & $0.00 \%$ & $0.00 \%$ & $0.00 \%$ & $0.08 \%$ & $0.03 \%$ & $0.13 \%$ & $0.02 \%$ & $0.01 \%$ & $0.03 \%$ \\
\hline Asia, South & $0.00 \%$ & $0.00 \%$ & $0.00 \%$ & $0.00 \%$ & $0.00 \%$ & $0.01 \%$ & $0.00 \%$ & $0.00 \%$ & $0.00 \%$ \\
\hline Asia, Southeast & $0.01 \%$ & $0.01 \%$ & $0.02 \%$ & $0.03 \%$ & $0.01 \%$ & $0.04 \%$ & $0.00 \%$ & $0.00 \%$ & $0.00 \%$ \\
\hline Australasia & $0.47 \%$ & $0.13 \%$ & $0.81 \%$ & $0.48 \%$ & $0.15 \%$ & $0.81 \%$ & $0.20 \%$ & $0.04 \%$ & $0.36 \%$ \\
\hline Caribbean & $0.36 \%$ & $0.18 \%$ & $0.54 \%$ & $0.07 \%$ & $0.04 \%$ & $0.11 \%$ & $0.01 \%$ & $0.00 \%$ & $0.01 \%$ \\
\hline Europe, Central & $1.07 \%$ & $0.34 \%$ & $1.81 \%$ & $1.15 \%$ & $0.36 \%$ & $1.94 \%$ & $0.10 \%$ & $0.00 \%$ & $0.25 \%$ \\
\hline Europe, Eastern & $0.10 \%$ & $0.02 \%$ & $0.19 \%$ & $0.09 \%$ & $0.02 \%$ & $0.16 \%$ & $0.02 \%$ & $0.00 \%$ & $0.04 \%$ \\
\hline Europe, Western & $0.63 \%$ & $0.13 \%$ & $1.13 \%$ & $1.24 \%$ & $0.39 \%$ & $2.08 \%$ & $0.44 \%$ & $0.08 \%$ & $0.80 \%$ \\
\hline $\begin{array}{l}\text { Latin America, } \\
\text { Andean }\end{array}$ & $0.26 \%$ & $0.05 \%$ & $0.47 \%$ & $0.14 \%$ & $0.03 \%$ & $0.24 \%$ & $0.08 \%$ & $0.00 \%$ & $0.16 \%$ \\
\hline $\begin{array}{l}\text { Latin America, } \\
\text { Central }\end{array}$ & $0.49 \%$ & $0.21 \%$ & $0.78 \%$ & $0.32 \%$ & $0.15 \%$ & $0.48 \%$ & $0.04 \%$ & $0.02 \%$ & $0.05 \%$ \\
\hline $\begin{array}{l}\text { Latin America, } \\
\text { Southern }\end{array}$ & $0.47 \%$ & $0.13 \%$ & $0.80 \%$ & $1.12 \%$ & $0.31 \%$ & $1.92 \%$ & $0.34 \%$ & $0.09 \%$ & $0.59 \%$ \\
\hline $\begin{array}{l}\text { Latin America, } \\
\text { Tropical }\end{array}$ & $0.83 \%$ & $0.38 \%$ & $1.28 \%$ & $1.08 \%$ & $0.35 \%$ & $1.81 \%$ & $0.09 \%$ & $0.04 \%$ & $0.15 \%$ \\
\hline $\begin{array}{l}\text { Northern Africa / } \\
\text { Middle East }\end{array}$ & $0.01 \%$ & $0.01 \%$ & $0.02 \%$ & $0.00 \%$ & $0.00 \%$ & $0.01 \%$ & $0.00 \%$ & $0.00 \%$ & $0.00 \%$ \\
\hline $\begin{array}{l}\text { North America } \\
\text { [High Income] }\end{array}$ & $0.80 \%$ & $0.30 \%$ & $1.31 \%$ & $0.60 \%$ & $0.21 \%$ & $0.98 \%$ & $0.13 \%$ & $0.02 \%$ & $0.24 \%$ \\
\hline Oceania & $0.00 \%$ & $0.00 \%$ & $0.00 \%$ & $0.00 \%$ & $0.00 \%$ & $0.00 \%$ & $0.00 \%$ & $0.00 \%$ & $0.00 \%$ \\
\hline $\begin{array}{l}\text { Sub-Saharan } \\
\text { Africa, Central }\end{array}$ & $0.01 \%$ & $0.00 \%$ & $0.01 \%$ & $0.01 \%$ & $0.00 \%$ & $0.02 \%$ & $0.00 \%$ & $0.00 \%$ & $0.00 \%$ \\
\hline $\begin{array}{l}\text { Sub-Saharan } \\
\text { Africa, East }\end{array}$ & $0.03 \%$ & $0.02 \%$ & $0.05 \%$ & $0.07 \%$ & $0.03 \%$ & $0.11 \%$ & $0.03 \%$ & $0.01 \%$ & $0.04 \%$ \\
\hline $\begin{array}{l}\text { Sub-Saharan } \\
\text { Africa, Southern }\end{array}$ & $0.11 \%$ & $0.05 \%$ & $0.18 \%$ & $0.12 \%$ & $0.06 \%$ & $0.18 \%$ & $0.03 \%$ & $0.02 \%$ & $0.05 \%$ \\
\hline $\begin{array}{l}\text { Sub-Saharan } \\
\text { Africa, } \\
\text { Western }\end{array}$ & $0.05 \%$ & $0.02 \%$ & $0.08 \%$ & $0.11 \%$ & $0.05 \%$ & $0.17 \%$ & $0.03 \%$ & $0.02 \%$ & $0.05 \%$ \\
\hline
\end{tabular}




\section{Appendix Table 4a}

Deaths attributable to HIV/AIDS from not adhering to antiretroviral therapy because of heavy alcohol consumption by Global Burden of Disease region for 2004 (Men)

\begin{tabular}{|c|c|c|c|c|c|c|c|c|c|}
\hline \multirow[b]{2}{*}{$\begin{array}{l}\text { Global Burden of } \\
\text { Disease region }\end{array}$} & \multicolumn{3}{|c|}{ 15-34 years } & \multicolumn{3}{|c|}{ 35-64 years } & \multicolumn{3}{|c|}{$65+$ years } \\
\hline & $\begin{array}{c}\text { Point } \\
\text { estimate }\end{array}$ & $\begin{array}{c}\text { Lower } \\
\text { estimate }\end{array}$ & $\begin{array}{c}\text { Upper } \\
\text { estimate }\end{array}$ & $\begin{array}{c}\text { Point } \\
\text { estimate }\end{array}$ & $\begin{array}{c}\text { Lower } \\
\text { estimate }\end{array}$ & $\begin{array}{c}\begin{array}{c}\text { Upper } \\
\text { estimate }\end{array} \\
\end{array}$ & $\begin{array}{c}\begin{array}{c}\text { Point } \\
\text { estimate }\end{array} \\
\end{array}$ & $\begin{array}{c}\text { Lower } \\
\text { estimate }\end{array}$ & $\begin{array}{c}\begin{array}{c}\text { Upper } \\
\text { estimate }\end{array} \\
\end{array}$ \\
\hline $\begin{array}{l}\text { Asia, Pacific } \\
\text { [High Income] }\end{array}$ & 0 & 0 & 1 & 2 & 1 & 4 & 0 & 0 & 0 \\
\hline Asia, Central & 0 & 0 & 0 & 0 & 0 & 0 & 0 & 0 & 0 \\
\hline Asia, East & 2 & 1 & 3 & 29 & 10 & 47 & 0 & 0 & 0 \\
\hline Asia, South & 6 & 3 & 10 & 12 & 5 & 19 & 0 & 0 & 0 \\
\hline Asia, Southeast & 22 & 11 & 32 & 84 & 37 & 130 & 1 & 0 & 1 \\
\hline Australasia & 0 & 0 & 0 & 1 & 0 & 1 & 0 & 0 & 0 \\
\hline Caribbean & 12 & 5 & 20 & 33 & 14 & 53 & 1 & 0 & 1 \\
\hline Europe, Central & 2 & 1 & 4 & 4 & 2 & 6 & 0 & 0 & 0 \\
\hline Europe, Eastern & 11 & 3 & 19 & 14 & 4 & 23 & 0 & 0 & 0 \\
\hline Europe, Western & 15 & 6 & 25 & 57 & 22 & 91 & 3 & 1 & 5 \\
\hline $\begin{array}{l}\text { Latin America, } \\
\text { Andean }\end{array}$ & 8 & 2 & 13 & 16 & 6 & 27 & 0 & 0 & 0 \\
\hline $\begin{array}{l}\text { Latin America, } \\
\text { Central }\end{array}$ & 48 & 19 & 78 & 85 & 37 & 133 & 2 & 1 & 3 \\
\hline $\begin{array}{l}\text { Latin America, } \\
\text { Southern }\end{array}$ & 8 & 3 & 13 & 16 & 6 & 26 & 1 & 0 & 1 \\
\hline $\begin{array}{l}\text { Latin America, } \\
\text { Tropical }\end{array}$ & 78 & 35 & 121 & 125 & 47 & 202 & 1 & 0 & 2 \\
\hline $\begin{array}{l}\text { Northern Africa / } \\
\text { Middle East }\end{array}$ & 1 & 0 & 1 & 1 & 0 & 1 & 0 & 0 & 0 \\
\hline $\begin{array}{l}\text { North America } \\
\text { [High Income] }\end{array}$ & 31 & 13 & 50 & 90 & 37 & 144 & 3 & 1 & 5 \\
\hline Oceania & 0 & 0 & 0 & 0 & 0 & 0 & 0 & 0 & 0 \\
\hline $\begin{array}{l}\text { Sub-Saharan } \\
\text { Africa, Central }\end{array}$ & 1 & 1 & 2 & 4 & 1 & 6 & 0 & 0 & 0 \\
\hline $\begin{array}{l}\text { Sub-Saharan } \\
\text { Africa, East }\end{array}$ & 51 & 22 & 80 & 245 & 104 & 387 & 5 & 2 & 7 \\
\hline $\begin{array}{l}\text { Sub-Saharan } \\
\text { Africa, Southern }\end{array}$ & 183 & 64 & 303 & 412 & 145 & 680 & 5 & 2 & 7 \\
\hline $\begin{array}{l}\text { Sub-Saharan } \\
\text { Africa, Western }\end{array}$ & 34 & 13 & 55 & 131 & 52 & 209 & 2 & 1 & 2 \\
\hline World & 515 & 202 & 829 & 1,360 & 530 & 2,190 & 23 & 9 & 37 \\
\hline
\end{tabular}




\section{Appendix Table 4b}

Deaths attributable to HIV/AIDS from not adhering to antiretroviral therapy because of heavy alcohol consumption by Global Burden of Disease region for 2004 (Women)

\begin{tabular}{|c|c|c|c|c|c|c|c|c|c|}
\hline \multirow[b]{2}{*}{$\begin{array}{l}\text { Global Burden of } \\
\text { Disease region }\end{array}$} & \multicolumn{3}{|c|}{ 15-34 years } & \multicolumn{3}{|c|}{ 35-64 years } & \multicolumn{3}{|c|}{$65+$ years } \\
\hline & $\begin{array}{c}\text { Point } \\
\text { estimate }\end{array}$ & $\begin{array}{c}\text { Lower } \\
\text { estimate }\end{array}$ & $\begin{array}{c}\text { Upper } \\
\text { estimate }\end{array}$ & $\begin{array}{c}\begin{array}{c}\text { Point } \\
\text { estimate }\end{array} \\
\end{array}$ & $\begin{array}{c}\text { Lower } \\
\text { estimate }\end{array}$ & $\begin{array}{c}\text { Upper } \\
\text { estimate }\end{array}$ & $\begin{array}{c}\text { Point } \\
\text { estimate }\end{array}$ & $\begin{array}{c}\text { Lower } \\
\text { estimate }\end{array}$ & $\begin{array}{c}\begin{array}{c}\text { Upper } \\
\text { estimate }\end{array} \\
\end{array}$ \\
\hline $\begin{array}{l}\text { Asia, Pacific } \\
\text { [High Income] }\end{array}$ & 0 & 0 & 0 & 0 & 0 & 0 & 0 & 0 & 0 \\
\hline Asia, Central & 0 & 0 & 0 & 0 & 0 & 0 & 0 & 0 & 0 \\
\hline Asia, East & 0 & 0 & 0 & 2 & 1 & 3 & 0 & 0 & 0 \\
\hline Asia, South & 0 & 0 & 0 & 0 & 0 & 1 & 0 & 0 & 0 \\
\hline Asia, Southeast & 1 & 1 & 2 & 3 & 1 & 4 & 0 & 0 & 0 \\
\hline Australasia & 0 & 0 & 0 & 0 & 0 & 0 & 0 & 0 & 0 \\
\hline Caribbean & 8 & 4 & 12 & 2 & 1 & 3 & 0 & 0 & 0 \\
\hline Europe, Central & 1 & 0 & 2 & 1 & 0 & 1 & 0 & 0 & 0 \\
\hline Europe, Eastern & 2 & 0 & 4 & 2 & 0 & 4 & 0 & 0 & 0 \\
\hline Europe, Western & 2 & 0 & 4 & 9 & 3 & 16 & 0 & 0 & 1 \\
\hline $\begin{array}{l}\text { Latin America, } \\
\text { Andean }\end{array}$ & 1 & 0 & 3 & 1 & 0 & 1 & 0 & 0 & 0 \\
\hline $\begin{array}{l}\text { Latin America, } \\
\text { Central }\end{array}$ & 9 & 4 & 14 & 6 & 3 & 9 & 0 & 0 & 0 \\
\hline $\begin{array}{l}\text { Latin America, } \\
\text { Southern }\end{array}$ & 1 & 0 & 2 & 3 & 1 & 5 & 0 & 0 & 0 \\
\hline $\begin{array}{l}\text { Latin America, } \\
\text { Tropical }\end{array}$ & 18 & 8 & 28 & 37 & 12 & 63 & 0 & 0 & 0 \\
\hline $\begin{array}{l}\text { Northern Africa / } \\
\text { Middle East }\end{array}$ & 0 & 0 & 0 & 0 & 0 & 0 & 0 & 0 & 0 \\
\hline $\begin{array}{l}\text { North America } \\
\text { [High Income] }\end{array}$ & 7 & 3 & 12 & 16 & 6 & 26 & 0 & 0 & 0 \\
\hline Oceania & 0 & 0 & 0 & 0 & 0 & 0 & 0 & 0 & 0 \\
\hline $\begin{array}{l}\text { Sub-Saharan } \\
\text { Africa, Central }\end{array}$ & 2 & 1 & 3 & 3 & 1 & 5 & 0 & 0 & 0 \\
\hline $\begin{array}{l}\text { Sub-Saharan } \\
\text { Africa, East }\end{array}$ & 43 & 20 & 66 & 145 & 68 & 223 & 1 & 1 & 2 \\
\hline $\begin{array}{l}\text { Sub-Saharan } \\
\text { Africa, Southern }\end{array}$ & 116 & 47 & 185 & 180 & 87 & 273 & 1 & 1 & 2 \\
\hline $\begin{array}{l}\text { Sub-Saharan } \\
\text { Africa, Western }\end{array}$ & 30 & 13 & 46 & 92 & 40 & 144 & 1 & 0 & 1 \\
\hline World & 242 & 102 & 381 & 502 & 224 & 781 & 4 & 2 & 6 \\
\hline
\end{tabular}




\section{Appendix Table 5a}

Potential years of life lost attributable to HIV from not adhering to antiretroviral therapy because of heavy alcohol consumption by Global Burden of Disease region for 2004 (Men)

\begin{tabular}{|c|c|c|c|c|c|c|c|c|c|}
\hline \multirow[b]{2}{*}{$\begin{array}{l}\text { Global Burden of } \\
\text { Disease region }\end{array}$} & \multicolumn{3}{|c|}{ 15-34 years } & \multicolumn{3}{|c|}{ 35-64 years } & \multicolumn{3}{|c|}{$65+$ years } \\
\hline & $\begin{array}{c}\text { Point } \\
\text { estimate }\end{array}$ & $\begin{array}{c}\text { Lower } \\
\text { estimate }\end{array}$ & $\begin{array}{c}\text { Upper } \\
\text { estimate }\end{array}$ & $\begin{array}{c}\text { Point } \\
\text { estimate }\end{array}$ & $\begin{array}{c}\text { Lower } \\
\text { estimate }\end{array}$ & $\begin{array}{c}\text { Upper } \\
\text { estimate }\end{array}$ & $\begin{array}{c}\text { Point } \\
\text { estimate }\end{array}$ & $\begin{array}{c}\text { Lower } \\
\text { estimate }\end{array}$ & $\begin{array}{c}\text { Upper } \\
\text { estimate }\end{array}$ \\
\hline $\begin{array}{l}\text { Asia, Pacific } \\
\text { [High Income] }\end{array}$ & 13 & 5 & 21 & 48 & 19 & 76 & 1 & 0 & 1 \\
\hline Asia, Central & 8 & 2 & 13 & 6 & 2 & 10 & 0 & 0 & 0 \\
\hline Asia, East & 51 & 15 & 87 & 613 & 208 & 1,017 & 2 & 1 & 3 \\
\hline Asia, South & 195 & 86 & 304 & 266 & 117 & 416 & 1 & 0 & 1 \\
\hline Asia, Southeast & 642 & 328 & 955 & 1,786 & 785 & 2,787 & 5 & 2 & 7 \\
\hline Australasia & 4 & 1 & 7 & 15 & 6 & 25 & 1 & 0 & 1 \\
\hline Caribbean & 348 & 147 & 548 & 694 & 287 & 1,101 & 5 & 1 & 8 \\
\hline Europe, Central & 72 & 27 & 117 & 81 & 32 & 130 & 1 & 1 & 2 \\
\hline Europe, Eastern & 328 & 98 & 557 & 285 & 84 & 485 & 1 & 0 & 2 \\
\hline Europe, Western & 393 & 141 & 645 & 1,169 & 461 & 1,878 & 22 & 8 & 37 \\
\hline $\begin{array}{l}\text { Latin America, } \\
\text { Andean }\end{array}$ & 227 & 69 & 384 & 346 & 123 & 569 & 0 & 0 & 0 \\
\hline $\begin{array}{l}\text { Latin America, } \\
\text { Central }\end{array}$ & 1,423 & 556 & 2,290 & 1,814 & 786 & 2,841 & 14 & 6 & 23 \\
\hline $\begin{array}{l}\text { Latin America, } \\
\text { Southern }\end{array}$ & 223 & 83 & 363 & 345 & 126 & 564 & 5 & 2 & 9 \\
\hline $\begin{array}{l}\text { Latin America, } \\
\text { Tropical }\end{array}$ & 2,199 & 987 & 3,410 & 2,670 & 1,013 & 4,327 & 10 & 3 & 17 \\
\hline $\begin{array}{l}\text { Northern Africa / } \\
\text { Middle East }\end{array}$ & 18 & 9 & 28 & 12 & 6 & 17 & 0 & 0 & 0 \\
\hline $\begin{array}{l}\text { North America } \\
\text { [High Income] }\end{array}$ & 799 & 329 & 1,269 & 1,715 & 695 & 2,734 & 25 & 9 & 40 \\
\hline Oceania & 1 & 0 & 2 & 2 & 0 & 5 & 0 & 0 & 0 \\
\hline $\begin{array}{l}\text { Sub-Saharan } \\
\text { Africa, Central }\end{array}$ & 41 & 15 & 66 & 75 & 28 & 123 & 0 & 0 & 0 \\
\hline $\begin{array}{l}\text { Sub-Saharan } \\
\text { Africa, East }\end{array}$ & 1,406 & 612 & 2,199 & 5,065 & 2,149 & 7,982 & 43 & 20 & 65 \\
\hline $\begin{array}{l}\text { Sub-Saharan } \\
\text { Africa, Southern }\end{array}$ & 4,994 & 1,737 & 8,251 & 8,616 & 3,032 & 14,199 & 41 & 18 & 63 \\
\hline $\begin{array}{l}\text { Sub-Saharan } \\
\text { Africa, Western }\end{array}$ & 949 & 364 & 1,534 & 2,695 & 1,073 & 4,317 & 14 & 6 & 21 \\
\hline World & 14,331 & 5,612 & 23,050 & 28,318 & 11,032 & 45,603 & 190 & 78 & 301 \\
\hline
\end{tabular}




\section{Appendix Table 5b}

Potential years of life lost attributable to HIV from not adhering to antiretroviral therapy because of heavy alcohol consumption by Global Burden of Disease region for 2004 (Women)

\begin{tabular}{|c|c|c|c|c|c|c|c|c|c|}
\hline \multirow[b]{2}{*}{$\begin{array}{l}\text { Global Burden of } \\
\text { Disease region }\end{array}$} & \multicolumn{3}{|c|}{ 15-34 years } & \multicolumn{3}{|c|}{ 35-64 years } & \multicolumn{3}{|c|}{$65+$ years } \\
\hline & $\begin{array}{c}\begin{array}{c}\text { Point } \\
\text { estimate }\end{array} \\
\end{array}$ & $\begin{array}{c}\text { Lower } \\
\text { estimate }\end{array}$ & $\begin{array}{c}\begin{array}{c}\text { Upper } \\
\text { estimate }\end{array} \\
\end{array}$ & $\begin{array}{c}\begin{array}{c}\text { Point } \\
\text { estimate }\end{array} \\
\end{array}$ & $\begin{array}{c}\text { Lower } \\
\text { estimate }\end{array}$ & $\begin{array}{c}\begin{array}{c}\text { Upper } \\
\text { estimate }\end{array} \\
\end{array}$ & $\begin{array}{c}\text { Point } \\
\text { estimate }\end{array}$ & $\begin{array}{c}\text { Lower } \\
\text { estimate }\end{array}$ & $\begin{array}{c}\begin{array}{c}\text { Upper } \\
\text { estimate }\end{array} \\
\end{array}$ \\
\hline $\begin{array}{l}\text { Asia, Pacific } \\
\text { [High Income] }\end{array}$ & 1 & 0 & 2 & 2 & 1 & 4 & 0 & 0 & 0 \\
\hline Asia, Central & 0 & 0 & 1 & 1 & 0 & 1 & 0 & 0 & 0 \\
\hline Asia, East & 1 & 0 & 1 & 38 & 14 & 62 & 0 & 0 & 0 \\
\hline Asia, South & 2 & 1 & 3 & 10 & 4 & 16 & 0 & 0 & 0 \\
\hline Asia, Southeast & 34 & 17 & 50 & 63 & 32 & 94 & 0 & 0 & 0 \\
\hline Australasia & 1 & 0 & 1 & 1 & 0 & 1 & 0 & 0 & 0 \\
\hline Caribbean & 236 & 117 & 355 & 47 & 24 & 69 & 0 & 0 & 0 \\
\hline Europe, Central & 32 & 10 & 54 & 15 & 5 & 25 & 0 & 0 & 0 \\
\hline Europe, Eastern & 67 & 13 & 121 & 45 & 9 & 81 & 0 & 0 & 0 \\
\hline Europe, Western & 61 & 12 & 109 & 206 & 65 & 347 & 2 & 0 & 4 \\
\hline $\begin{array}{l}\text { Latin America, } \\
\text { Andean }\end{array}$ & 43 & 8 & 78 & 17 & 4 & 30 & 0 & 0 & 0 \\
\hline $\begin{array}{l}\text { Latin America, } \\
\text { Central }\end{array}$ & 264 & 112 & 416 & 131 & 61 & 200 & 0 & 0 & 0 \\
\hline $\begin{array}{l}\text { Latin America, } \\
\text { Southern }\end{array}$ & 27 & 8 & 47 & 70 & 20 & 119 & 0 & 0 & 1 \\
\hline $\begin{array}{l}\text { Latin America, } \\
\text { Tropical }\end{array}$ & 522 & 237 & 807 & 822 & 268 & 1,375 & 1 & 0 & 2 \\
\hline $\begin{array}{l}\text { Northern Africa / } \\
\text { Middle East }\end{array}$ & 1 & 1 & 2 & 0 & 0 & 1 & 0 & 0 & 0 \\
\hline $\begin{array}{l}\text { North America } \\
\text { [High Income] }\end{array}$ & 194 & 73 & 316 & 325 & 117 & 534 & 2 & 0 & 3 \\
\hline Oceania & 0 & 0 & 0 & 0 & 0 & 0 & 0 & 0 & 0 \\
\hline $\begin{array}{l}\text { Sub-Saharan } \\
\text { Africa, Central }\end{array}$ & 47 & 19 & 75 & 70 & 27 & 113 & 0 & 0 & 0 \\
\hline $\begin{array}{l}\text { Sub-Saharan } \\
\text { Africa, East }\end{array}$ & 1,259 & 594 & 1,925 & 3,294 & 1,534 & 5,053 & 11 & 5 & 17 \\
\hline $\begin{array}{l}\text { Sub-Saharan } \\
\text { Africa, Southern }\end{array}$ & 3,353 & 1,362 & 5,345 & 4,069 & 1,960 & 6,178 & 12 & 6 & 17 \\
\hline $\begin{array}{l}\text { Sub-Saharan } \\
\text { Africa, Western }\end{array}$ & 862 & 378 & 1,346 & 2,070 & 892 & 3,249 & 6 & 3 & 10 \\
\hline World & 7,008 & 2,962 & 11,054 & 11,294 & 5,037 & 17,552 & 35 & 16 & 54 \\
\hline
\end{tabular}




\section{Appendix Figure 1}

Deaths attributable to HIV/AIDS from not adhering to antiretroviral therapy by Global Burden of Disease region for 2004 per 10,000,000 people 15 years or older

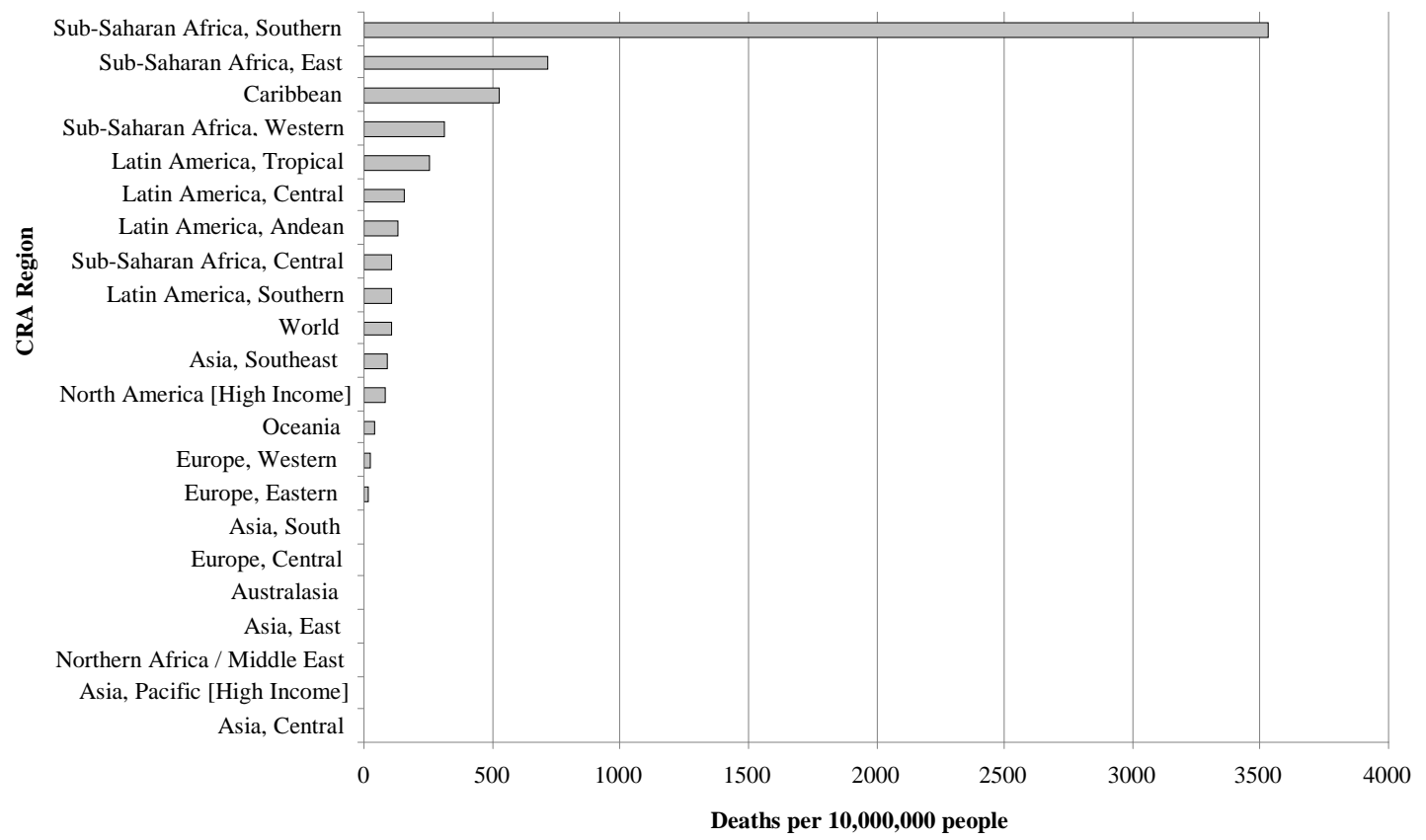

Appendix Figure 2

Potential years of life lost attributable to HIV/AIDS from not adhering to antiretroviral therapy by Global Burden of Disease region for 2004 per 10,000,000 people 15 years or older

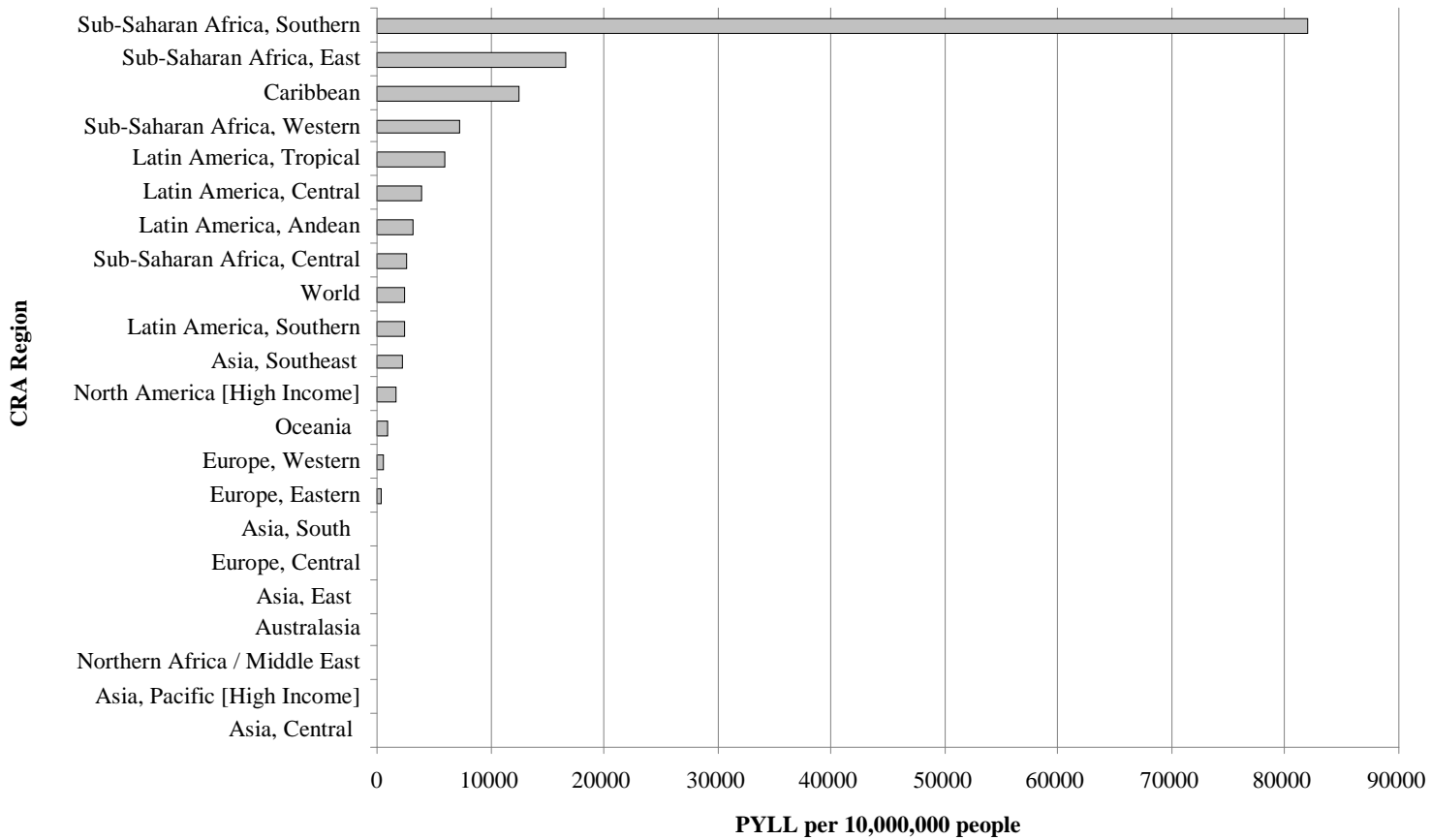

